\title{
1 Rapid evolution and horizontal gene transfer in the genome of a male-killing Wolbachia
}

2 Tom Hill ${ }^{1}$, Robert L. Unckless ${ }^{1} \&$ Jessamyn I. Perlmutter ${ }^{1 *}$

3 1. 4055 Haworth Hall, The Department of Molecular Biosciences, University of Kansas, 1200 Sunnyside

4 Ave, Lawrence, KS, 66045.

5

$6 \quad *$ Corresponding author: jessamyn.perlmutter@ku.edu

7 Keywords: Wolbachia, Drosophila innubila, male killing, genome evolution, phage WO 


\section{Abstract}

Wolbachia are widespread bacterial endosymbionts that infect a large proportion of insect species. While some strains of this bacteria do not cause observable host phenotypes, many strains of Wolbachia have some striking effects on their hosts. In some cases, these symbionts manipulate host reproduction to increase the fitness of infected, transmitting females. Here we examine the genome and population genomics of a male-killing Wolbachia strain, wInn, that infects Drosophila innubila mushroom-feeding flies. We compared wInn to other closely-related Wolbachia genomes to understand the evolutionary dynamics of specific genes. The $w$ Inn genome is similar in overall gene content to $w \mathrm{Mel}$, but also contains many unique genes and repetitive elements that indicate distinct gene transfers between wInn and nonDrosophila hosts. We also find that genes in the Wolbachia prophage and Octomom regions are particularly rapidly evolving, including those putatively or empirically confirmed to be involved in host pathogenicity. Of the genes that rapidly evolve, many also show evidence of recent horizontal transfer among Wolbachia symbiont genomes, suggesting frequent movement of rapidly evolving regions among individuals. These dynamics of rapid evolution and horizontal gene transfer across the genomes of several Wolbachia strains and divergent host species may be important underlying factors in Wolbachia's global success as a symbiont.

\section{Introduction}

Wolbachia are the most widespread endosymbionts on the planet, infecting an estimated 40-52\% of all insect species (ZUG AND HAMMERSTEIN 2012; WEINERT et al. 2015). These obligate intracellular Gram-negative $\alpha$-proteobacteria of the order Rickettsiales infect the gonads of their hosts and are primarily transmitted vertically via the cytoplasm from mother to offspring (HERTIG AND WOLBACH 1924; SERBUS AND SULLIVAN 2007). Wolbachia of insects and other arthropods have adopted cunning techniques to facilitate their matrilineal spread by manipulating host reproduction to increase the proportion of infected, transmitting females in the population (WERREN et al. 2008; HURST AND FROST 2015). The most common form of this reproductive parasitism is cytoplasmic incompatibility $(\mathrm{CI})$, where crosses between infected males and uninfected females result in death of offspring. If the mother is also infected with a compatible strain, offspring are rescued from death, giving infected females a fitness advantage in the population over uninfected females (Yen AND BARR 1971; TURELli AND HOFFMAN 1991; SinKINS et al. 1995). Three other, less common forms of reproductive parasitism rely on sex ratio distortion to increase the proportion of transmitting females each generation. These phenotypes are known as parthenogenesis (asexual reproduction of females, (RUSSELL AND STOUTHAMER 2011)), feminization (genetic males physically develop and reproduce as females, (BOUCHON et al. 1998; KAGEYAMA et al. 2002)), and male killing (infected males die, (HURST et al. 1999; DYSON et al. 2002)). Additional forms of transmission, including horizontal transfer between hosts of different species or strains (VAVRE et al. 1999; HAINE et al. 2005), are 
less understood and thought to be comparatively rare, but are likely key to Wolbachia's ubiquitous spread around the world (SANAEI et al. 2020).

The incredible success of Wolbachia in becoming one of the world's most widespread infections (WERREN et al. 2008; LEPAGE AND BORDENSTEIN 2013) is in part due to its diverse genetic toolkit. Indeed, Wolbachia strains are so diverse that they are divided into many supergroup clades, of which 18 have been named (TAYLOR et al. 2018; LAIDOUDI et al. 2020; LEFOULON et al. 2020). Studies on strains in supergroups A and B are the best represented in the literature, and include many reproductive parasite strains of hosts such as mosquitoes and a large number of Drosophila species (GERTH et al. 2014). Much of the diversity between Wolbachia genomes is contributed from prophage WO, the genome of phage WO of Wolbachia that has inserted itself into the bacterial chromosome and replicates along with core Wolbachia genes. Prophage WO sometimes retains the potential to form infective phage particles later, and sometimes degrades over time losing the potential to form new viral particles (METCALF et al. 2014; BORDENSTEIN AND BORDENSTEIN 2016). Prophages and phages are highly mobile and dynamic elements in the genome, often picking up new genes via horizontal gene transfer, and many such unique prophage WO genes have the potential to confer important functions in interactions with the eukaryotic host (BORDENSTEIN AND BORDENSTEIN 2016). Indeed, functional and evolutionary analyses of the genetic loci that underlie CI have shown that they are in fact prophage WO genes that interact directly with the eukaryotic host to manipulate reproductive and developmental processes (BECKMANN et al. 2017; LEPAGE et al. 2017; LINDSEY et al. 2018; BECKMANN et al. 2019). Related to phage WO is a cassette of 8 genes known as Octomom. This cassette contains paralogs of phage WO genes but replicates separately and copy number of Octomom regions is correlated with regulation of Wolbachia titer, making strains more or less pathogenic to the host (CHROSTEK AND TEIXEIRA 2015; DUARTE et al. 2020).

Despite the great diversity and interest in a variety of Wolbachia infections, most research attention has focused on CI, largely due to its use in vector control strategies around the globe (ZABALOU et al. 2004). These programs take advantage of the natural abilities of Wolbachia to both block viral transmission and spread itself via reproductive parasitism (HEDGES et al. 2008; TEIXEIRA et al. 2008; O'NEILL et al. 2018; MAINS et al. 2019; RoSS et al. 2019). Comparatively fewer analyses have been done on Wolbachia genomes of strains that induce male killing (DYER AND JAENIKE 2004; DuPlOUY et al. 2013; METCALF et al. 2014). However, male killing merits additional analysis due to its potential in vector control (BEREC et al. 2016), role in shaping arthropod evolution (JIGGINS et al. 2000), and the close relationship between CI and male killing (DYER et al. 2005). Indeed, the CI genetic loci are located only a few genes away from the malekilling candidate gene, wmk (WO-mediated killing) in the Wolbachia strain of Drosophila melanogaster ( w Mel) (PERLMUTTER et al. 2019). Also, many male-killing and CI strains are closely related (SHEELEY AND MCALLISTER 2009), and several strains are multipotent in that they can switch between the two 
phenotypes either within the same host or between different hosts (HURST et al. 2000; SASAKI et al. 2002; JAENIKE 2007). The close genetic relationship between the two phenotypes indicates that studies on male killing may inform CI and vice versa. In addition, their overall similarities may help narrow down evolutionary dynamics that are unique to each phenotype or shared between them.

Among the few Wolbachia male-killers of flies is the strain infecting Drosophila innubila, wInn (DYER AND JAENIKE 2004). This strain is particularly interesting as it is closely related to Wolbachia found in the main Drosophila model species, wMel of D. melanogaster, which causes CI (SHEELEY AND MCALLISTER 2009). In addition, the symbiosis between wInn and its host has been maintained for thousands of years, and despite this, there is no evidence of host resistance to the phenotype in modern populations (JAENIKE AND DYER 2008; UNCKLESS AND JAENIKE 2012). Due to the close relationship between $w \mathrm{Mel}$ and $w \mathrm{Inn}$ and the longstanding symbiosis of $w \mathrm{Inn}$ with its host, analysis of $w$ Inn population dynamics can be used to uncover evolutionary trends that may be important in reproductive parasitism generally, male-killers specifically, or other interactions with the host.

Here, we sequence the genome of the Wolbachia strain infecting D. innubila, wInn, and conduct population genomic analyses using sequences from 48 Wolbachia-infected individual wild females from Wolbachia's ability to rapidly adapt to diverse hosts. Finally, we examine population structure and coinheritance of Wolbachia with mitochondria to determine if horizontal transmission occurs frequently in wInn.

Methods

\section{Genome sequence of $w$ Inn}

For a single Wolbachia-positive strain described previously (UNCKLESS AND JAENIKE 2012), we extracted DNA following the protocol described in (CHAKRABORTY et al. 2017). We prepared the DNA as a sequencing library using the Oxford Nanopore Technologies Rapid 48-h (SQK-RAD002) protocol, which was then sequenced using a MinION (Oxford Nanopore Technologies, Oxford, UK; NCBI SRA: TBD) (JAIN et al. 2016). The same DNA was also used to construct a fragment library with insert sizes of $\sim 180 \mathrm{bp}$, and we sequenced this library on an Illumina HiSeq 4000 (150 bp paired-end, Illumina, San Diego, CA, NCBI SRA: TBD).

Oxford Nanopore sequencing read bases were called post hoc using the built in 
the raw Oxford Nanopore sequencing reads alongside the Illumina paired-end short sequencing reads using SPAdes version 3.13.0 (BANKEVICH et al. 2012), which generated an initial assembly of 83 contigs. We then attempted to improve this initial assembly using the 83 assembled fragments, along with Nanopore sequencing reads and Illumina paired-end short sequencing reads in MaSuRCA version 3.4.1 (ZIMIN et al. 2013), defining the expected genome size as 1.5 million bp. This produced a single contig $1,286,799 \mathrm{bp}$ long. We then used Pilon version 1.23 to polish the genome with minion fragments for 3 iterations (WALKER et al. 2014) and further polished with Racon version 1.4.3 for three iterations using the short read data (VASER et al. 2017). We then verified the contiguity of the assembly using BUSCO version 3.0 (SIMÃO et al. 2015). From a search for 221 proteobacteria orthologs, we found 181 complete single copy orthologs and 2 fragmented orthologs (compared to 180 complete and 2 fragmented for the published $w$ Mel genome: NC_002978.6).

\section{Fly collections and Wolbachia infection confirmation}

We collected wild Drosophila at four mountainous locations across Arizona between the 22nd of August and the 11th of September 2017 (HILl AND UNCKLESS 2020b; HILL AND UNCKLESS 2020a). Specifically, we collected at the Southwest research station in the Chiricahua mountains (31.871 latitude, 109.237 longitude), Prescott National Forest (34.540 latitude, -112.469 longitude), Madera Canyon in the Santa Rita mountains (31.729 latitude, -110.881 longitude) and Miller Peak in the Huachuca mountains bisporus) placed in large piles about $30 \mathrm{~cm}$ in diameter, at least 5 baits per location. A sweep net was used to collect flies over the baits in either the early morning or late afternoon between one and three days after the bait was left. Flies were sorted by sex and our best guess of species based on morphology at the University of Arizona and were flash frozen at $-80^{\circ} \mathrm{C}$ before being shipped on dry ice to Lawrence, KS. Specifically, we separated individuals likely to be Drosophila innubila from the rest of the collections for further processing and genetic confirmation of species identification.

We further analyzed the 343 putative $D$. innubila flies which we homogenized and extracted DNA from using the Qiagen Gentra Puregene Tissue kit (USA Qiagen Inc., Germantown, MD, USA) (HILL AND UNCKLESS 2020b; HILL AND UNCKLESS 2020a). We tested these samples for infection using Wolbachia primers specific to the Wolbachia surface protein (wsp) gene alongside a positive and negative control (ZHOU et al. 1998). $\mu \mathrm{l}$ of $20 \mathrm{mM} \mathrm{MgCl} 2$ (Solis Biodyne), $1 \mu \mathrm{l}$ of dNTPs (20 $\mu \mathrm{M}$ each), $0.5 \mu \mathrm{l}$ of forward (F) primer (81F 5'TGGTCCAATAAGTGATGAAGAAAC-3', $20 \mu \mathrm{M}$ ), $0.5 \mu \mathrm{l}$ of reverse (R) primer (691R 5'AAAAATTAAACGCTACTCCA-3', $20 \mu \mathrm{M}$ ), $0.5 \mu \mathrm{l}$ of Taq DNA polymerase (5 U/ $\mu \mathrm{l}$ ) (Solis Biodyne) and water to make up the final volume of $10 \mu \mathrm{l}$. The amplification reaction consisted of one cycle of $1 \mathrm{~min}$ 
at $94^{\circ} \mathrm{C}, 1 \mathrm{~min}$ at $58^{\circ} \mathrm{C}$ and $2 \mathrm{~min}$ at $72^{\circ} \mathrm{C}$, followed by 35 cycles of $15 \mathrm{~s}$ at $94^{\circ} \mathrm{C}, 1 \mathrm{~min}$ at $58^{\circ} \mathrm{C}$ and $2 \mathrm{~min}$ at $72^{\circ} \mathrm{C}$, and one cycle of $15 \mathrm{~s}$ at $94^{\circ} \mathrm{C}, 1 \mathrm{~min}$ at $58^{\circ} \mathrm{C}$ and $7 \mathrm{~min}$ at $72^{\circ} \mathrm{C}$. These conditions yielded 610 basepair (bp) PCR products, which we observed running out the product on a 1\% agarose TAE gel. This survey yielded 48 Wolbachia-positive lines.

For the 48 Wolbachia-infected lines we previously extracted DNA and sequenced the host and Wolbachia genomes on two runs of an Illumina HiSeq 4000 (150 bp paired-end (HILL AND UNCKLESS 2020a; HILL AND UNCKLESS 2020b), Illumina, San Diego, CA), producing an average of 20,618,752 reads per sample, of which an average of 436,527 mapped to Wolbachia per sample, as summarized in Supplementary Table 1.

\section{Genome annotation}

We annotated the $w$ Inn genome using Prokka version 1.15.4 (SEEMANN 2014), detecting 2686 total genes, of which 1390 were retained following size and quality filtering (> 50bp, quality score > 20, Supplementary Table 2). Using this annotation of the genome, we extracted coding sequences and generated amino acid sequences using GFFread version 0.12.1 (PERTEA 2011). We also downloaded the coding sequence and amino acid sequences for open reading frames in the Wolbachia of Drosophila melanogaster (Canton S strain) (wMel-CS, SAMN02604000), the Wolbachia of Drosophila simulans (Riverside strain) (wRi, SAMN02603205), the Wolbachia of Drosophila simulans (Hawaii strain) (wHa, SAMN02604273), and the Wolbachia of Culex pipiens (wPip, SAMN02296948). We used blastp version 2.9.0 (ALTSCHUL et al. 1990) to identify orthologs for these genes in $w \operatorname{Inn}$ (parameters: hsp = 1, num_alignments = 1, e-value $<0.00001$ ). For each set of orthologs we created a gene alignment using MAFFT version 7.409 (parameters: --auto) and for 100 randomly chosen genes made a visual inspection of amino acid sequences to confirm similarity of putative orthologous sequences. We then verified the completeness of the extracted amino acids sequences using BUSCO version 3.0 (SIMÃO et al. 2015). From a search for 221 proteobacteria orthologs, we found 185 complete single copy orthologs and 1 fragmented ortholog, and 1 complete and duplicated ortholog (compared to 184 complete, 1 duplicated and 2 fragmented for the published $w$ Mel genome: NC_002978.6).

To annotate the repetitive content of the wInn genome, we used RepeatModeler version 2.0.1 (SMIT AND HUBLEY 2008) and RepeatMasker version 4.0.9 (parameters: -gff -gccalc -s -norna) (SMIT AND HUBLEY 2013-2015).

\section{Genomic variation in wInn}

For all 48 Wolbachia-positive lines collected in 2017, we mapped short reads to the D. innubila genome (HILL et al. 2019), masked using RepeatMasker version 4.0.9 (parameters: -gff -gccalc -pa 4 -s) (SMIT AND HUBLEY 2013-2015), a custom library of D. innubila repeats (HILl et al. 2019), and the masked wInn genome using BWA MEM version 0.7.17-r1188 (LI AND DURBIN 2009) and SAMtools version 1.9 
(LI et al. 2009). We then extracted aligned reads mapping to $w$ Inn and used GATK version 4.0.0 to remove optical and PCR duplicates and realign around indels (MCKENNA et al. 2010; DEPRISTO et al. 2011). We then called variants in the wInn genome of each Wolbachia-positive lines using GATK HaplotypeCaller version 4.0.0 (MCKENNA et al. 2010; DEPRISTO et al. 2011), considering only variants with a quality score greater than 500. Finally, we combined VCFs using BCFtools version 1.7 (NARASIMHAN et al. 2016) to create a multiple sample VCF.

\section{Detection of selection on Wolbachia genes}

For each $w$ Inn gene with an ortholog in $w \mathrm{Ha}$ and $w \mathrm{Ri}$, we generated an alignment of the coding sequence of each gene using MAFFT version 7.409 (parameters: --auto). Following this alignment, we reformatted the alignment into a PAML version 1.3.1 usable format and generated a gene tree using PRANK version 0.170427 (parameters: $+\mathrm{F}$-showtree $-\mathrm{d}=$ =paml) (LÖYTYNOJA 2014). We next used codeML (YANG 2007 ) to calculate the non-synonymous divergence $(\mathrm{dN})$ and synonymous divergence (dS) across the entire gene tree and find the best fitting branches model (Model 7 or 8), as well as calculate $\mathrm{dN}$ and $\mathrm{dS}$ on each branch of the tree (Model 1), specifically looking at the estimates of $\mathrm{dN} / \mathrm{dS}$ on the $w$ Inn branch versus all other branches. For both the total tree and specifically the wInn branch, we looked for gene functional categories with higher dN/dS than all other genes, after controlling for gene length.

\section{Population structure across $w$ Inn populations}

For synonymous sites in the VCF, we used VCFtools version 0.1.16 (DANECEK et al. 2011) to calculate the fixation index $\left(\mathrm{F}_{\mathrm{st}}\right)$ between each population and the other populations (BROWN 1970). We also performed a principle component analysis on the variation found across the samples in $\mathrm{R}$ version 3.5.1 (TEAM 2013), using the VCF input as a presence/absence matrix.

\section{Ancient and recent horizontal transfer}

We reasoned that if no horizontal gene transfer was occurring, then Wolbachia variation would be perfectly linked to mitochondrial variation, while non-vertical transfer would break that pattern. To assess this, we looked at all pairwise combinations of mitochondrial and Wolbachia alleles and recorded loci with all four allele sets across the two loci across the 48 samples (e.g. GT, AT, GC and AC), giving a recombination like signature (suggesting non-vertical inheritance). We then counted the number of discordant and non-discordant SNPs in 10-kbp windows across the wInn genome to identify specific sections enriched for discordant SNPs. We used a $\chi^{2}$ test to identify specific functional categories enriched for discordant SNPs.

For long term horizontal gene transfer, we used the VHICA R package version 0.2.7 to calculate $\mathrm{dS}$ vs codon bias for all pairwise for all shared genes for pairwise combinations of $w$ Inn, $w \mathrm{Ha}$ and $w \mathrm{Ri}$ (WALLAU et al. 2016). We reasoned that horizontal transfer of a gene from a highly divergent Wolbachia 
would produce a signal of increased dS between $w \mathrm{Ha}$ and $w \mathrm{Inn}$ for that gene and could polarize which species had the horizontal transfer event based on the $\mathrm{dS}$ of that gene in the pairs $w \mathrm{Inn}-w \mathrm{Ha}, w \mathrm{Ha}-w \mathrm{Ri}$ and $w$ Inn- $w \mathrm{Ri}$. We considered $\mathrm{dS}$ to be excessively high in a gene if it was greater than the mean $\mathrm{dS}+$ the variance for that window of effective number of codons ( 5 codons window size, sliding 5 codons) (WALLAU et al. 2016). We considered a gene to be a putative horizontal acquisition in $w$ Inn if dS compared to $w H a$ and $w \mathrm{Ri}$ is excessively high compared, but $\mathrm{dS}$ is also not significantly higher when comparing $w \mathrm{Ha}$ to $w \mathrm{Ri}$. We then performed a $\chi^{2}$ test to look for functional categories that are enriched for putatively horizontally acquired genes.

Finally, we assessed the extent of ancient horizontal transfer across the Wolbachia phylogeny. We downloaded all Wolbachia genomes and their annotations from the NCBI genome database (summarized in Supplementary Table 1), based on the known NCBI annotations we found groups of orthologous genes.

221 We generated codon alignments for these orthologous genes using MAFFT (parameters: --auto) (KATOH et al. 2002), and generated a gene tree for each gene using PhyML (model $=$ GTR, gamma $=8$, bootstraps = 100) (GUINDON et al. 2010). We also generated a whole species phylogeny for these genomes and to generated a multigene phylogeny with 100 bootstraps using PhyML ( model $=$ GTR, gamma $=8$, bootstraps suggest little discordance between phylogenies). Finally, we performed a $\chi^{2}$ test to look for functional categories that are enriched for putatively horizontally transferred genes.

Results

\section{$w$ Inn genome assembly reveals a genome similar to $w$ Mel and evidence of horizontal gene transfer}

D. innubila is a mycophagous species in the Drosophila subgenus, found throughout the southwestern USA and northwestern Mexico on mountain-top forests known as 'Sky Islands', separated by large expanses of desert (JAENIKE et al. 2003; DYER AND JAENIKE 2005; DYER et al. 2005; JAENIKE AND DYER 2008). Since the Wolbachia strain of Drosophila innubila (wInn) is one of the few Wolbachia strains known to cause male killing in Drosophila (JAENIKE et al. 2003; DYER AND JAENIKE 2005), studying its evolutionary and population dynamics allows new genetic insights into male-killing populations. To that end, we examined the genome and population genomic variation in $w$ Inn. In a previous survey we collected wild D. innubila from four geographically isolated mountain locations and tested strains for Wolbachia using PCR to amplify wsp primers and found 48 lines infected with Wolbachia (Supplementary Table 1, 13 
from the Chiricahua mountains, 27 from Prescott, 2 from the Huachucas, and 6 from the Santa Ritas) (HILL AND UNCKLESS 2020b; HILL AND UNCKLESS 2020a).

We sequenced and assembled the genome using a combination of short and long reads for one strain. The $w$ Inn genome is a single chromosome 1,247,635 base pairs long, with $35.1 \%$ GC content (Figure 1A). We found 1390 genes, 1331 found previously in other Wolbachia: 1292 genes are shared with wMel, 1248 shared with $w$ Rec, and 1059 shared with $w$ Ri. We found the $w$ Inn genome had a BUSCO score of 81.9\% (181 complete single copy orthologs and 2 fragmented orthologs, from a sample of 221) compared to $81.3 \%$ in $w \mathrm{Mel}$ (180 complete single copy orthologs and 2 fragmented orthologs, from a sample of 221). Of the 1331 previously identified genes, 954 are conserved across all four genomes (Figure 1C, Supplementary Table 2), including 12 prophage WO-A and 54 prophage WO-B genes in all genomes (Supplementary Table 3), and 9 Octomom genes (5 orthologs to $w \mathrm{Mel}$ and 4 paralogs of these, genes linked to Wolbachia pathogenicity) (CHROSTEK AND TEIXEIRA 2015). Interestingly, these Octomom genes are not found in a single cassette like in $w \mathrm{Mel}$ but are instead spread throughout the genome (Figure 1A). The genes orthologous to WO-B genes are found in 3 groups (Figure 1A, called WOInn-B1, WOInn-B2 and WOInnB3). Despite the fragmentation of the prophage regions, the genes are syntenic to the prophage-B region in $w$ Mel. We also found 10 Type IV secretion pump genes, found in two cassettes, as in $w$ Mel (Figure 1A). Consistent with previous results, $w$ Inn is closely related to $w \mathrm{Mel}$ within supergroup A, clustering with other supergroup A Wolbachia genomes (Figure 1B, Supplementary Figure 1).

We found 3 genes are shared between the male-killing $w$ Inn genome and $w$ Rec (which reportedly kills males when introgressed into a sister species, but causes CI in its native host (JAENIKE 2007)), but absent in the $w \mathrm{Mel}$ and $w \mathrm{Ri}$ genomes, both of which induce $\mathrm{CI}$ in their native hosts (Figure 1C). All three genes are hypothetical proteins found in other non-male-killing Wolbachia supergroup A genomes (including other varieties of $w \mathrm{Mel}$ ). These genes are therefore not likely to be involved with male-killing specifically, but have been gained in the ancestral supergroup A, then lost in $w$ Mel-CS and $w$ Ri genomes. $w$ Inn does not appear to have a reduced, relic prophage genome like $w$ Rec, and instead shares most prophage genes with $w \mathrm{Mel}$ despite the more distant phylogenetic relationship (Figure 1C, (METCALF et al. 2014)). The 57 genes absent in $w$ Rec but present in $w$ Inn and $w$ Mel consists of 21 prophage genes, 4 transcription genes, 9 metabolism genes, and 21 genes of unknown function. We attempted to further confirm the differences in genomic content by mapping short reads from $w$ Rec, $w \mathrm{Mel}, w \operatorname{Inn}$ and $w \mathrm{Ha}$ to each of the genomes pairwise and find the exact same number of genes shared in each case, supporting the

274 the regions orthologous to $0.35-0.55 \mathrm{Mb}$ and $1.24-1.28 \mathrm{Mb}$ in the $w \mathrm{Inn}$ genome, which also includes a large 275 portion of the prophage WO-B genome. 
Of the 59 coding sequences in wInn, but not found in other Wolbachia, 33 of these have high similarity to mRNA in Formica wood ants that may have an overlapping range with $D$. innubila (nonredundant megablast e-value < 0.00005) (FRANCOEUR 1973; AlTSCHUL et al. 1990). D. innubila also contains multiple transposable element (TE) sequences shared with Camponotus (a genus within Formica) (HILL et al. 2019), and so horizontal transfer may occur frequently between these species. Mites are a potential vector for horizontal transfer of genes and Wolbachia (HOUCK et al. 1993; BROWN AND LLOYD 2015) and in keeping with this, we find 7 new wInn genes have a high similarity to Varroa destructor transcriptome sequences (non-redundant megablast e-value $<0.00005$, Supplementary Table 3). Though this is not a common Drosophila mite, it may be closely related to other Drosophila mites without sequenced genomes that may vector genes between Wolbachia strains. Alternatively, it may be a source of other Wolbachia infections in D. innubila, or horizontal exchange of genes between Wolbachia strains (BROWN AND LLOYD 2015). These genes could also be undescribed mobile elements which have spread from a different species, like the mobile elements described below. The nine remaining sequences have no known orthologs. Among the 157 genes absent in $w$ Inn but present in $w$ Mel, we found no functional categories enriched ( $p$-value > 0.12).

We found $12.38 \%$ of the wInn genome is repetitive, similar to other Wolbachia (Figure 1A) (FOSTER et al. 2005; WOOLFIT et al. 2013); most of these sequences are short simple repeats, satellites, and insertions from 16 bacterial insertion sequences (selfish elements found in bacteria). $1.49 \%$ of the genome consists of insertions of a single hAT family element (hobo-like DNA transposon found in Drosophila, rnd1_family-6) inserted in 14 loci across the genome and 3.74\% consists of 3 LINE elements inserted in 37 loci across the genome (long interspersed nuclear elements, an RNA transposon order found in Drosophila), primarily in clusters (Figure 1A) (WICKER et al. 2007). Consistent with the potential horizontal transfer seen for several genes, we find one LINE element (rnd-1_family-12 with 14 insertions) is homologous to a LINE found previously in Varroa destructor (or a close relative), while another (rnd-1_family-165 with 12 insertions) is homologous to a LINE found in Formica wood ants (non-redundant megablast e-value < 0.00005) (ALTSCHUL et al. 1990). No homologous sequence can be identified for the hAT element (nonredundant megablast e-value $=1$ no hits), which is also the only TE found as complete sequences suggesting a more recent horizontal acquisition of full active copies, while most of the LINE element insertions are degraded, supporting ancient horizontal acquisitions (Supplementary Figure 2).

Figure 1. A. Genome schematic of the wInn genome. Circles correspond to the following: 1) GC content of the wInn genome in 10-kbp windows, between 30\% and 40\%. Darker colors have higher GC content. 2) Locations of genes thought to interact with hosts, specifically prophage orthologous to WO-A and WO-B genes in $w \mathrm{Mel}$ (blue), Type IV secretion pumps (green), and Octomom genes (yellow). 3) Loci of nonphage genes. 4) Loci of repetitive content, with short simple repeats and interspersed satellites (SSR and 
IS, black), and hAT or LINE TE insertions (red). B. Phylogeny of Wolbachia genomes closely related to wInn for reference, as a subset of Supplementary Figure 1. Bootstrap support of each branch is shown on the nodes (of 100 bootstraps). A description of each Wolbachia genome and the species they infect is given in Supplementary Table 1. C. The overlap of genes between $w \mathrm{Inn}, w \mathrm{Mel}$, and $w$ Ri. D. Synteny between $w \mathrm{Mel}$ and $w \mathrm{Inn}$, with single large inversion shown in blue, while consistent synteny groups are shown in 315 grey.

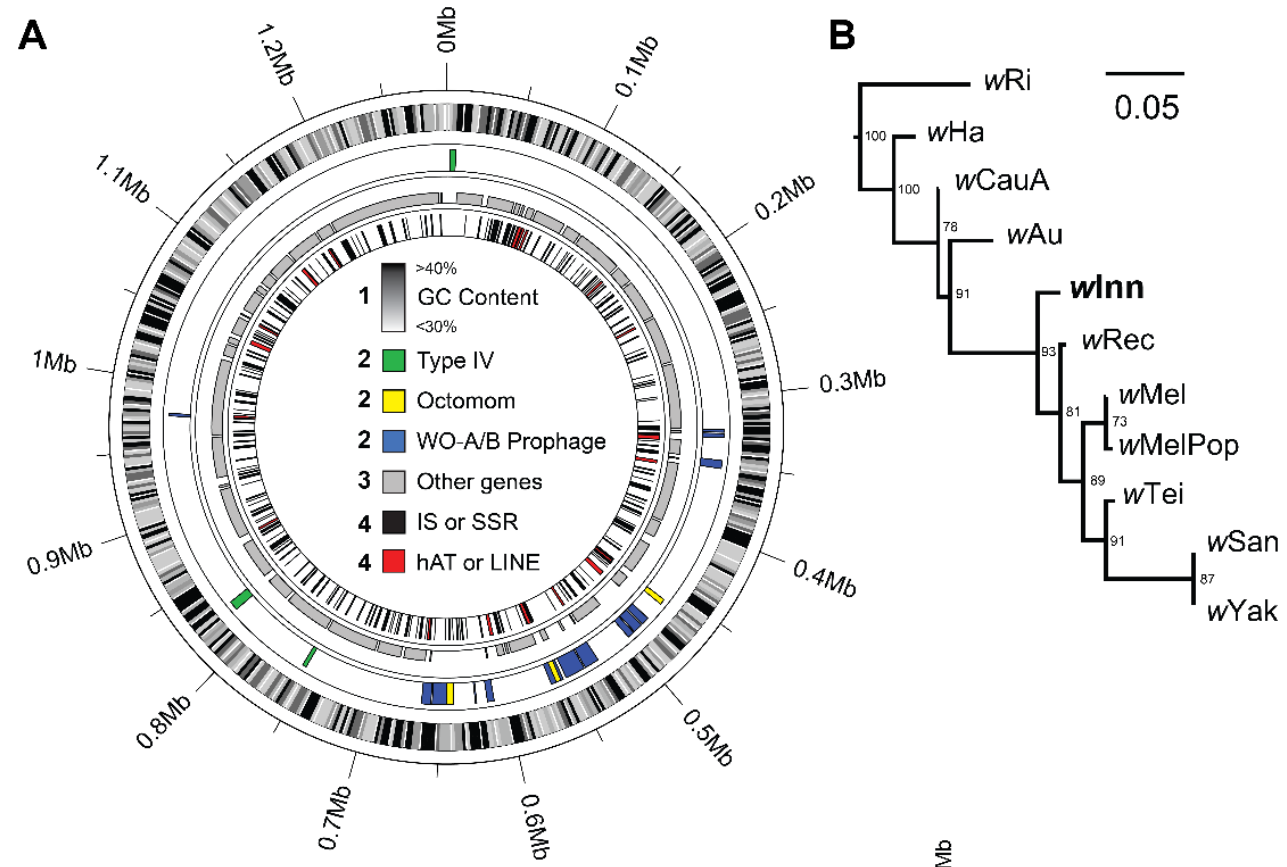

C
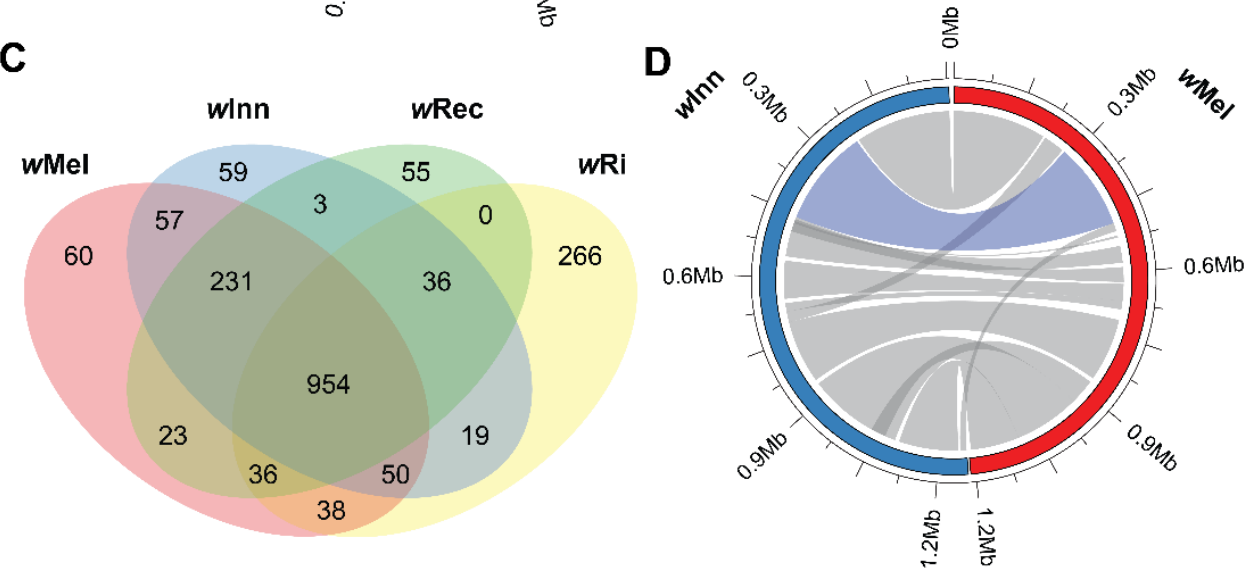

\section{Octomom and prophage genes are rapidly evolving in wInn and other Wolbachia}

318 We next used PAML to determine genes rapidly evolving in wInn compared to the closely related Wolbachia genomes (YANG 2007). For each ortholog set, we identified the proportion of synonymous (dS) substitutions and amino acid changing, nonsynonymous substitutions (dN) (per possible synonymous or nonsynonymous substitution, respectively) occurring on each branch of the phylogeny to identify changes between the gene sequence of $w \mathrm{Inn}, w \mathrm{Ha}$, and $w \mathrm{Ri}$. We expect $\mathrm{dN} / \mathrm{dS}$ to be higher when genes are faster 
evolving, due to more nonsynonymous fixations, potentially due to positive selection (YANG 2007). We chose $w \mathrm{Ha}$ and $w \mathrm{Ri}$ over $w \mathrm{Mel}$ or other genomes as these genomes are diverged enough from $w$ Inn to provide some signal (unlike $w \mathrm{Mel}$ or $w \operatorname{Rec}$, where $\mathrm{dS} \sim 0.001$ between genomes), while not being diverged enough to have too little similarity or saturated rates of dS. Genes previously suspected to be involved in

327 pathogenicity (certain Octomom and prophage genes), are rapidly evolving on all branches (Figure 2A, 328 GLM t-value $=2.750, p$-value $=0.0061)$, while DNA metabolism genes are more rapidly evolving 329 exclusively on the $w$ Inn branch, though not significantly (Figure 2A, GLM $t$-value $=1.868, p$-value $=$ 330 0.0622). The fastest evolving DNA metabolism genes in wInn are the non-phage genes WD1095 ( $r a d C$, a 331 DNA repair protein), WD0065 (a DNA binding protein), WD0057 (a host integration factor) and WD0752 332 (xerC, a recombinase).

Several genes have been previously implicated in reproductive parasitism in Wolbachia in Drosophila, so we specifically examined the evolution of these genes in wInn and the other genomes (wmk: WD0626, cifA: WD0631, cifB: WD0632). These genes are evolving faster than background rates in wInn $(\mathrm{dN} / \mathrm{dS}=0.27-1.56$ in $w$ Inn, versus $\mathrm{dN} / \mathrm{dS}$ background median $=0.225)$, though not significantly $(\mathrm{GLM} \mathrm{t}-$ value $=0.224, p$-value $=0.642$ ). Additionally, $w m k$ is only rapidly evolving on the $w \mathrm{Ha}$ and $w \mathrm{Ri}$ branches $(\mathrm{dN} / \mathrm{dS}=1.56)$, while cifB (a gene thought to be involved with cytoplasmic incompatibility) (LEPAGE $e t$ al. 2017), is only rapidly evolving in $w \operatorname{Inn}$ (Figure $2, \mathrm{WD} 0632, \mathrm{dN} / \mathrm{dS}=1.10$ ). We also examined the rate of evolution of specific codons in these genes but find no specific sites are driving this rapid evolution in

341 these putative host manipulation genes ( $p$-value >0.05). The Type IV secretion genes are also faster 342 evolving than the background across the total phylogeny, but not significantly (GLM t-value $=1.427, p$ 343 value $=0.154)$.

344 Figure 2. Rate of evolution of the $w \mathrm{Inn}$ branch versus evolution on the $w \mathrm{Ri} / w \mathrm{Ha}$ branches. Functional 345 categories of interest (DNA metabolism genes, prophage genes and Octomom genes) are highlighted by 346 different shapes and colors. Dashed lines show dN/dS $=1$ for both axes, while the dotted line shows where $347 \mathrm{dN} / \mathrm{dS}$ is equal on the axes. Genes of interest, either due to putative involvement in Wolbachia 348 pathogenicity, or due to high $\mathrm{dN} / \mathrm{dS}$ in $w$ Inn exclusively are named and labelled with a black outline to distinguish them. 


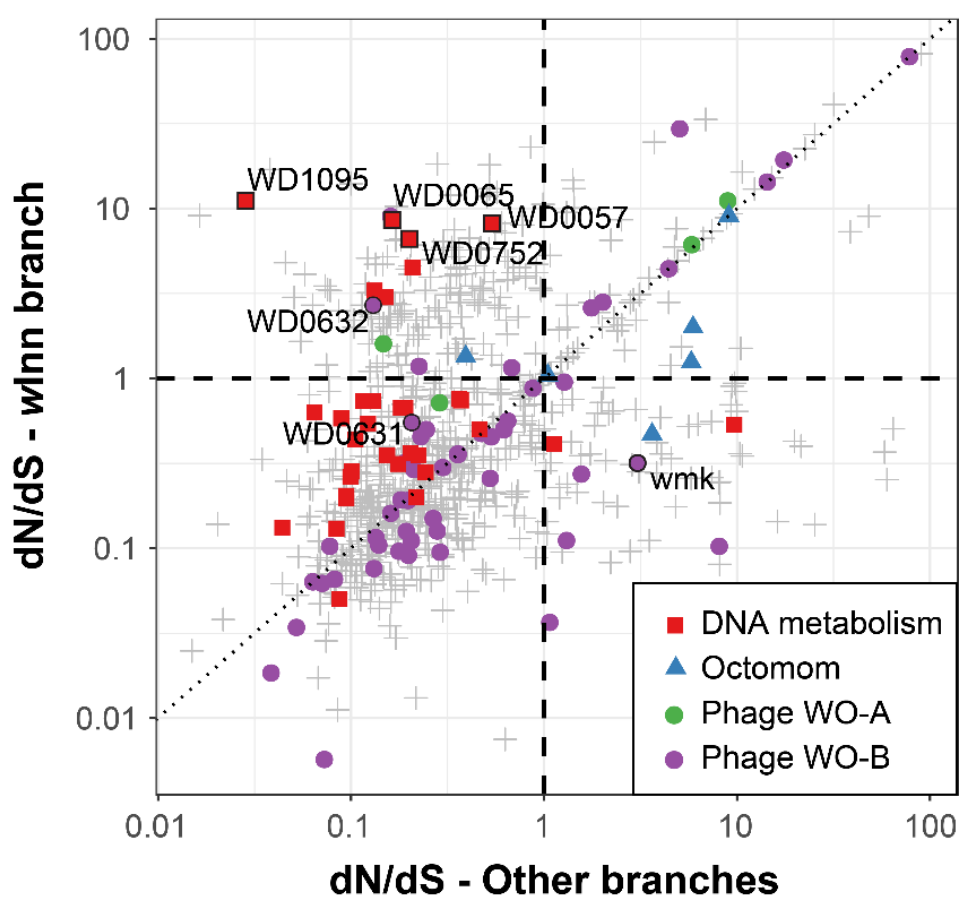

Some prophage genes show evidence of recent horizontal transfer in wInn and across the Wolbachia

\section{phylogeny}

Horizontal transfer is frequently occurring in microbes at different levels: symbiotic bacteria such as Wolbachia can switch hosts to propagate in new species and can switch between individual hosts within a species (VAVRE et al. 1999; HAINE et al. 2005; RIEGLER et al. 2005; WERREN et al. 2008; ILINSKY 2013).

Beyond this, specific genes and regions of genomes can horizontally transfer to and from bacteria of the same strain allowing for a recombination-like process which may facilitate adaptation, or can transfer to different strains which allows for the acquisition of new genes, allowing for adaptation to better propagate within their hosts, in a process known as horizontal gene transfer (LAWRENCE 1999; DUTTA AND PAN 2002).

We suspected that many symbiont genes potentially involved in unique wInn host-microbe interactions may be more likely to horizontally transfer between Wolbachia strains than other genes. So, we next looked for evidence of horizontal gene transfer since the divergence of $w$ Inn from $w \mathrm{Ha}$ and $w \mathrm{Ri}$. We used VHICA (WALLAU et al. 2016) to compare synonymous divergence (dS) to the effective number of codons for the pairwise comparisons of $w \mathrm{Inn}-w \mathrm{Ha}, w \mathrm{Inn}-w \mathrm{Ri}$, and $w \mathrm{Inn}-w \mathrm{Ha}$ for genes with orthologs in all three species. As dS is constrained by codon usage bias, it can be higher when the effective number of codons is higher, and so should be controlled for when comparing the divergence of two different genes (WALLAU et al. 2016). If a gene has horizontally transferred into wInn from another Wolbachia (but not $w \mathrm{Ha}$ or $w \mathrm{Ri}$ ), we expect the $\mathrm{dS}$ to be higher in these comparisons than expected, after controlling for codon usage bias (WALLAU et al. 2016). i.e. there will be elevated dS in both comparisons involving wInn, but 
not the $w \mathrm{Ha}-w \mathrm{Ri}$ comparison. We found 13 genes have elevated dS in just the $w$ Inn comparisons (Figure 3, Supplementary Table 4), suggesting potential horizontal gene acquisition from another Wolbachia. These genes are enriched for prophage WO-A \& WO-B genes $\left(\chi^{2}\right.$ test, $\chi^{2}=60.476, \mathrm{df}=1, p$-value $\left.=7.448 \mathrm{e}-15\right)$, and Octomom genes $\left(\chi^{2}\right.$ test, $\chi^{2}=181.64, \mathrm{df}=1, p$-value $\left.=2.2 \mathrm{e}-16\right)$ that have elevated divergence in $w$ Inn. The 6 genes not associated with the prophage or Octomom regions are all genes of unknown function. We did not suspect horizontal transfer of the Wolbachia organism to play a role in the elevated divergence seen here due to the similarity in $\mathrm{dS}$ for the $w \mathrm{Inn}-w \mathrm{Ri}$ and $w \mathrm{Ha}-w \mathrm{Ri}$ comparisons (Wilcoxon Rank Sum Test W $=462970, p$-value $=0.7687$ ).

Figure 3. Comparison of dS between pairs of Wolbachia suggesting horizontal transfer of genes. Point colors show the gene ontology categories (GO category) for Octomom genes and Prophage WO-B genes. Point shape indicates evidence of excessive divergence (and possible horizontal transfer) in either $w$ Inn, $w$ Ha, or both. Dashed lines show the mean + the variance for each axis as a vague cutoff for elevated synonymous divergence. Note this does not consider effective number of codons, so it is not the cutoff used to determine if there is elevated divergence (which is difficult to illustrate as it differs per gene), but it is a close approximation.

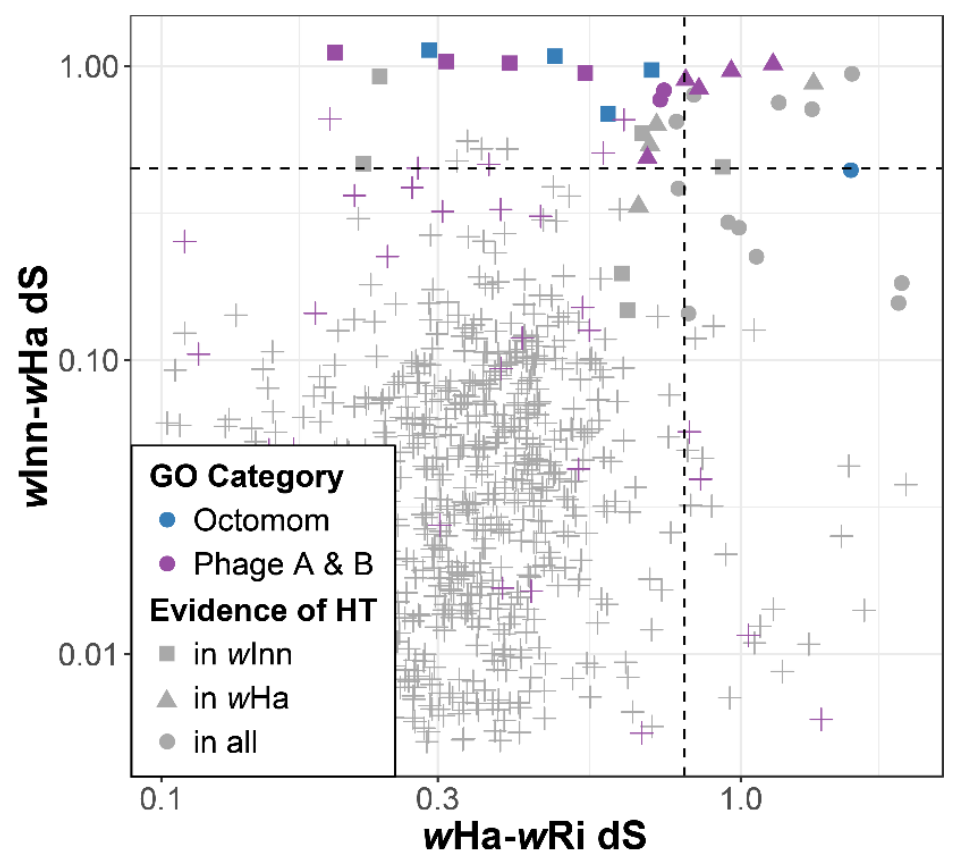

To examine if these gene categories frequently horizontally transfer, or if these transfers are unique to wInn, we downloaded 54 Wolbachia genomes (all genomes available for download on NCBI genomes, described in Supplementary Table 1) and made gene alignments for all orthologs and attempted to identify gene tree/species tree discordance. We assumed that excessive gene tree/species tree discordance would be due to large amounts of horizontal gene transfer. We attempted to look for functional categories which 
show more tree discordance than expected and across 847 orthologous genes, and found excessive amounts of discordance for prophage WO genes (Table 1, 36 of 47 genes, degrees of freedom $=1, \chi^{2}=111.1, p$ value $=5.62 \mathrm{e}-26)$ and Octomom genes $\left(\right.$ Table 1,7 of 7 genes, degrees of freedom $=1, \chi^{2}=71.27, p$-value

$395=3.395 \mathrm{e}-17)$ across large evolutionary distances, while no other categories have significantly more 396 discordance than expected. We find a significant overlap in the genes which have horizontally transferred 397 in $w$ Inn and across the whole phylogeny $\left(\chi^{2}=49.003, p\right.$-value $\left.=2.556 \mathrm{e}-12\right)$, suggesting that specific 398 (prophage) genes are more likely to horizontally transfer than others and demonstrating that the recent 399 horizontal transfers are not unique to this strain.

400 Table 1: Summary of species tree/gene tree discordance analysis. Using 847 orthologous genes across 54 401 genomes (Supplementary Table 5), we identified genes which showed significant discordance from the 402 species tree. Table shows the number of genes in each category showing discordance, and if this 403 discordance is significant using a $\chi^{2}$ test, using an expected number of discordant genes per category 404 based on the size of the category.

\begin{tabular}{ccccc}
\hline Functional Category & $\begin{array}{c}\text { Discordant } \\
\text { genes }\end{array}$ & $\begin{array}{c}\text { Genes in } \\
\text { category }\end{array}$ & $\chi^{\mathbf{2}}$ & $\boldsymbol{p}$-value \\
\hline Biosynthesis & 1 & 50 & 2.374 & 0.876 \\
Cell envelope & 1 & 31 & 0.952 & 0.671 \\
Cellular processes & 1 & 35 & 1.238 & 0.732 \\
DNA metabolism & 1 & 42 & 1.759 & 0.815 \\
Energy metabolism & 0 & 90 & 7.438 & 0.006 \\
Octomom & 7 & 7 & 71.278 & $3.395 \mathrm{e}-17$ \\
Phage WO-A & 12 & 13 & 111.105 & $5.626 \mathrm{e}-26$ \\
Phage WO-B & 24 & 33 & 165.927 & $5.817 \mathrm{e}-38$ \\
Protein synthesis & 1 & 144 & 9.985 & 0.002 \\
Regulatory functions & 0 & 11 & 0.909 & 0.340 \\
Transcription & 0 & 52 & 4.298 & 0.038 \\
Transport and binding & 0 & 44 & 3.636 & 0.057 \\
proteins & & & & \\
Type IV & 0 & 10 & 0.826 & 0.363 \\
Unknown function & 22 & 285 & 0.102 & 0.749 \\
\hline
\end{tabular}


We next mapped the short-read data for 48 samples of wild D. innubila infected with wInn, collected from four locations, to the repeat masked $w$ Inn genome and called polymorphism. From this, we identified 30 SNPs as coding synonymous, 69 SNPs as coding non-synonymous, and 235 SNPs as noncoding across all individuals. The wInn samples are highly structured based on both the total and synonymous variation (Figure 4). Using a principle component analysis, we find three clear clusters,

412 separating the Chiricahua and Prescott populations, and grouping the Santa Rita and the Huachuca populations together (Figure 4), as seen with the mitochondrial genome and consistent with previous

414 findings (JAENIKE et al. 2003; DYER AND JAENIKE 2005; JAENIKE AND DYER 2008; HILL AND UNCKLESS 2020a). This suggests that females are not moving between locations or at least not reproducing after moving, creating structure in the populations of this maternally inherited endosymbiont (JAENIKE et al. 2003).

Figure 4: Principal component analysis of genetic variation in wInn A. Map of locations samples were taken from in this survey, adapted from (HILL AND UNCKLESS 2020a). Phoenix and Tucson are shown as points of reference. B. total polymorphism and C. silent polymorphism in $w$ Inn samples, colored and shaped by location of collection, Chiricahuas ( $\mathrm{CH}$, red circles), Huachucas (HU, orange squares), Prescott 422 (PR, blue triangles) and Santa Ritas (SR, green diamonds).

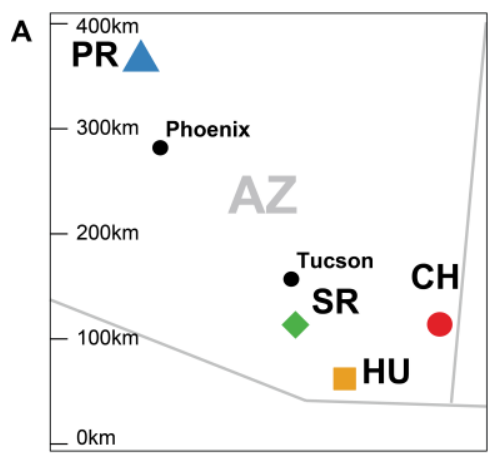

424

425

426

427

428

429

430

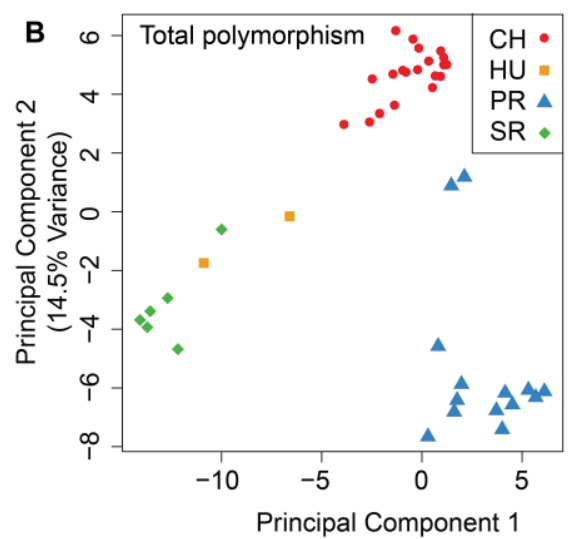

(27.1\% Variance)

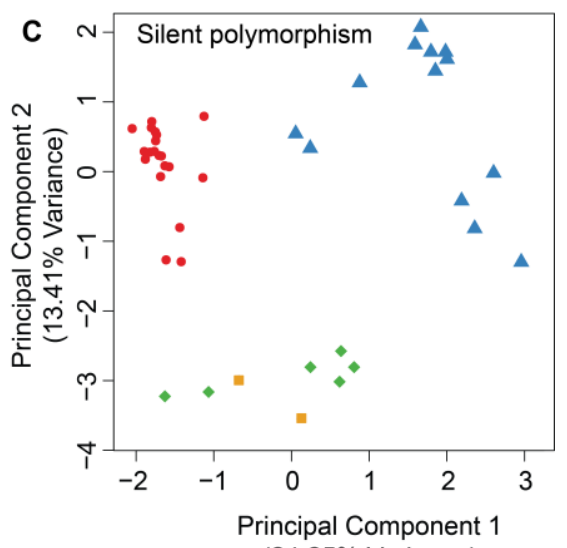

(24.25\% Variance)

However, when building a maximum-likelihood tree of the $w$ Inn samples using all polymorphisms in the core Wolbachia genes, we find some evidence of migration between populations (Supplementary Figure 3A). Specifically, we find two samples from $\mathrm{PR}$ cluster within $\mathrm{CH}$ and share the $\mathrm{CH}$ mitochondrial haplotype, suggesting there may be multiple Wolbachia types shared between population locations due to host migration (Supplementary Figure 3). These 2 PR samples are also closer to $\mathrm{CH}$ than other PR samples in the principal component analysis (Figure 4). This signature is not seen in the host, likely due to the recent establishment of D. innubila (HILL AND UNCKLESS 2020a), particularly in Prescott. 
To identify if specific factors are contributing to the population structure, we calculated the fixation

432 index $\left(\mathrm{F}_{\mathrm{ST}}\right)$, a measure of pairwise divergence between a subpopulation and the total population, between

433 the three clustered groups. We expect $F_{S T}$ to be elevated in cases where SNPs are found at high frequencies

434 in a single population but not the remaining samples. As expected with the non-recombining bacterial

435 genome, we found signatures of $F_{S T}$ are uniform genome wide, with no specific windows of elevated $F_{\text {ST }}$

436 compared to the rest of the genome ( $1 \mathrm{kbp}$ windows, GLM $p$-value > 0.432) and no functional categories

437 are enriched for high or low $\mathrm{F}_{\text {ST }}$ (Supplementary Table 6, GLM $p$-value > 0.611).

When comparing the inheritance of the maternally transmitted wInn and D. innubila mitochondria,

439 we found little evidence of discordant inheritance, consistent with a previous study (DYER AND JAENIKE

440 2005). We do however find evidence of three clusters of 49 SNPs in the wInn genome which show evidence

441 of recombination-like events (all four allele combinations between the Wolbachia site and the mitochondria

442 site, Supplementary Figure 4), suggesting either imperfect co-inheritance of the wInn and mitochondria,

443 recurrent mutation, or a horizontal transfer event. The two larger clusters are contained within the prophage

444 WO-A and WO-B portions of the genome, suggesting horizontal movement of the prophage is the cause of

445 this discordance. We also find the copy number of the prophage regions differs between wInn lines: the

446 prophage region has significantly higher sequence coverage in 8 of 48 lines, varying from 1-4x the average

447 coverage of the Wolbachia genome (Wilcoxon Rank Sum W > 443221, p-value < 0.04, Figure 5). In

448 addition, phage copy number is negatively correlated with Wolbachia titer. Indeed, higher phage coverage

449 correlates with lower Wolbachia titer up to a point, consistent with potentially active phage lysing symbiont

450 cells. This additional sequencing coverage of phage regions and negative correlation with titer despite a

451 potentially weaker signal from not controlling for host age or other factors may suggest that the prophage

452 region produces active phage particles. Active phage would also be consistent with horizontal transfer of

453 prophage genes between hosts, as discussed above (Figure 5).

454 Figure 5: Octomom copies compared to Wolbachia titer (total Wolbachia coverage/host autosomal

455 coverage) and prophage abundance genes (prophage coverage/total Wolbachia coverage) compared to the

456 Wolbachia titer (total Wolbachia coverage/host autosomal coverage). 


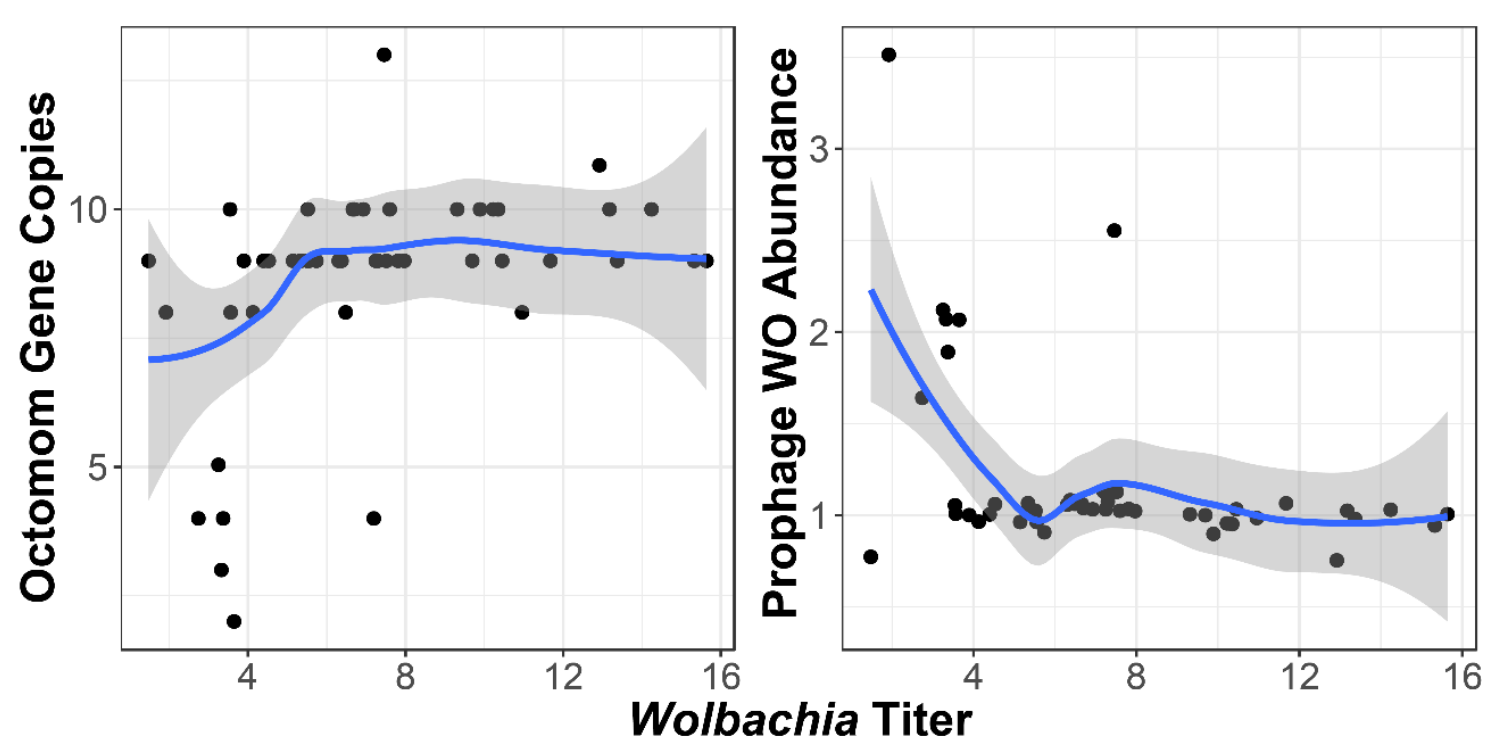

Given the rapid evolution of Octomom genes, and the previously identified association between increased pathogenicity and Octomom copy number, we also examined the effect of copy number on Wolbachia titer within lines. We found all strains contain Octomom genes in a cassette, though coverage of the Octomom genes appears to also differ between lines, consistent with potentially differing copy numbers of Octomom (Supplementary Figure 5). We also identified a positive correlation between Octomom copy number and Wolbachia titer, though it is not significant (Supplementary Figure 5, GLM tvalue $=1.930, p$-value $=0.0597)$. However, Wolbachia titer does not seem to be associated with Octomom copy number once Octomom copy number is greater than 9 (Supplementary Figure 5, GLM t-value $=0.534$, $p$-value $=0.596$ ). This suggests that while putatively increased Octomom copy number may be important for titer, it has a reduced effect higher than $\sim 8$ copies. The differences in titer are not significantly associated with any SNPs or gene duplications ( $p$-value > 0.186).

\section{Discussion}

We sequenced and assembled the genome of the Wolbachia strain infecting Drosophila innubila, wInn. $w$ Inn is one of the few strains of Wolbachia to cause male-killing in Drosophila (JAENIKE et al. 2003) and so we sought to examine the evolutionary dynamics of its genome, with particular focus on prophage and Octomom regions that have genes putatively or empirically involved in Wolbachia pathogenicity (MetCalF et al. 2014; ChrosteK AND TeIXeIRA 2015; Bordenstein AND Bordenstein 2016; BeCKMann et al. 2017; LePAge et al. 2017; PERLMUTTER et al. 2019). The genome content and dynamics of wInn are largely like other closely-related strains (Figure 1), sharing most of its genome with similar supergroup A Wolbachia strains. wRec is relatively closely related to $w$ Inn and has been reported to kill males when introgressed into a sister host species, D. subquinaria (JAENIKE 2007). When comparing the genomes of $w$ Inn and $w \operatorname{Rec}$ to the other closely related strains in this analysis (all CI-inducing), we identify 
only 3 unique genes. However, these genes are found in other Wolbachia strains not used in our initial analyses, based on the non-redundant BLAST database (ALTSCHUL et al. 1990; PRUITT et al. 2005). The lack of unique male-killer genes is consistent with the idea that male killing is often hidden. Indeed, many strains like $w$ Rec do not cause male killing in their native hosts but do kill males when transferred to other hosts or vice versa (FUJII et al. 2001; SASAKI et al. 2002; JAENIKE 2007; HUGHES AND RASGON 2014). In addition, there is evidence of host resistance that suppresses the phenotype in many systems, where the arms race between host and bacteria leads to loss of phenotype (HORNETT et al. 2006; JAENIKE 2007; MAJERUS AND MAJERUS 2010). These factors indicate that absence of phenotype does not necessarily correlate with absence of symbiont genotype and male killing is instead also heavily dependent on host background (i.e. male killing is not a simple matter of presence/absence of a male-killing toxin gene). The fact that the wmk male-killing gene candidate is found in many non-male killer genomes also supports the idea that male-killers do not necessarily have unique genetic content involved in the phenotype and a combination of host- and microbe-dependent factors are necessary for the phenotype to occur (PerlmutTer et al. 2019).

We also find that while the overall genetic content of $w$ Inn is similar to $w \mathrm{Mel}$, it has key differences most likely due to the loss of phage regions in $w \operatorname{Rec}$ (METCALF et al. 2014). It is intriguing to note that $w$ Rec contains relic phage regions that have lost many genes compared to those in $w \mathrm{Mel}$ and $w \mathrm{Inn}$, and is likely unable to produce viral particles because of the absence of key virus structural genes (METCALF et al. 2014). Meanwhile, $w$ Inn and $w \mathrm{Mel}$ have not (Figure 1). Both $w$ Inn and $w$ Rec are closely related, both are capable of male killing (JAENIKE et al. 2003; METCALF et al. 2014), and both infect mycophagous species, yet one has an eroded prophage region while the other does not (Figure 1). In addition, evidence here based on higher sequence coverage and the inverse relationship between phage titer and Wolbachia titer, further suggests that there may be active phage WO particle production (Supplementary Figure 5). It is unclear why $w$ Inn and $w$ Mel would putatively maintain viable phage particle production while $w$ Rec would not. Future research will be needed to determine any functional consequences of phage particles that may be playing a role in their retainment or loss across different systems.

We also identified 59 genes unique to the wInn genome, and most intriguingly, 33 of these are 510 there are several TEs with homology to those in Camponotus ants. Along with evidence of horizontal gene 511 transfer and rapid evolution, this homology with divergent hosts would suggest new possibilities for the 512 genetic transfer routes in this system. Indeed, mites are known to transfer Wolbachia infections among Drosophila populations (BROWN AND LLOYD 2015), and Varroa destructor is a common parasitic species 
found throughout the United States and the rest of the world (ROSENKRANZ et al. 2010). Therefore, it is possible that this or a similar species has vectored either the entire Wolbachia symbiont or some genes among various arthropods, contributing to horizontal gene transfer in this strain. Formica wood ants and Camponotus ants are also common across the United States (BOLTON et al. 2006), indicating that there is a possibility of interaction with the mites and/or $D$. innubila. The homology of $w$ Inn genes with ant genes may indicate that there has been an exchange of genes or symbionts among these hosts, possibly via mites, and the ants and mites in the area may contain similar strains. In this model, the mites would vector either genes or symbionts among interacting hosts (HOUCK et al. 1993; BROWN AND LLOYD 2015), and the active phage particles could also play a role by primarily or excessively moving the prophage region among hosts, which may be much easier and more common than symbiont exchange.

Horizontal transfer of genes between Wolbachia strains and hosts is in line with existing literature demonstrating Wolbachia's proclivity for genetic exchange. Indeed, the horizontal transfer of individual genes or the entire phage region among Wolbachia strains that is supported here reflects previously reported cases (WANG et al. 2016; COOPER et al. 2019). Phage WO genes appear to horizontally transfer between the Wolbachia genomes analyzed here more often than the rest of the genome, possibly due to the activity of the phage particles (Table 1, Figure 3), or the activity of surrounding mobile elements, as may have happened in the D. yakuba clade with horizontal transfer of the CI loci and nearby transposons (COOPER $e t$ al. 2019). Further, entire symbiont transfer may also potentially occur in this system, although it is rare if it does occur. Vertically transmitted symbionts, such as Wolbachia, are subject to strong selection within the host, and unlike frequently horizontally transferring microorganisms, can experience various degrees of genome reduction and other genetic adaptations that lead to essential ties with a specific host (MORAN 2002; DYER AND JAENIKE 2005; JAENIKE AND DYER 2008; MCCUTCHEON AND MORAN 2012). However, bypassing this phenomenon, there are Wolbachia strains that can transfer to new hosts via various modes of transmission and then sweep rapidly across a new and sometimes divergent host population (RIEGLER $e t$ al. 2005; BALDO et al. 2008; TURELLI et al. 2018; SANAEI et al. 2020). Whole symbiont transmission could be aided by frequent horizontal transfer of genes or entire regions, such as the prophage, just as we report

540 here in $w$ Inn. More broadly, acquisition of new genetic variants that the eukaryotic host is unfamiliar with

541 may confer an advantage that could allow the Wolbachia to be transferred to a new host or maintained in

542 an existing host. Indeed, some known cases of horizontal phage WO gene transfer among symbionts have 543 functional effects on the symbiont's ability to parasitize the host (WANG et al. 2016; COOPER et al. 2019).

544 Most crucially, horizontal gene transfer in Wolbachia is not restricted to exchange among phages or 545 bacteria, but also with the eukaryotic host. Phage WO stands unique among other described phages with its 546 regions of large genes containing eukaryotic-like domains that imply both lateral transfer between animal and phage WO genomes and potential interaction with the eukaryotic host (BORDENSTEIN AND 
BORDENSTEIN 2016). Among these genes in the prophage region known as the 'Eukaryotic Association Module' are the two CI loci and the male-killing gene candidate that empirically impact host reproductive biology (BECKMANn et al. 2017; LePAGE et al. 2017; PERLMUTTER et al. 2019). Thus, the acquisition of ant and mite genes in wInn reflects Wolbachia's unique genetic exchanges more broadly as well as its tripartite interactions among phages, bacteria, and animals. Elucidating the function and fitness impacts of these genes, if any, will be an interesting area of future research. Also, if there is indeed frequent horizontal exchange in this system, this may then be a driving force for maintenance of phage particles, as they may confer selective advantages over time. Further supporting the idea of recurrent genetic exchanges in the wInn ecosystem is evidence of both recently acquired TEs and more ancient, degraded TEs, some of which are homologous to those in Formica and Varroa.

Notably, we find a surprising amount of this repeat content in the wInn genome, like other Wolbachia (Figure 1A, Supplementary Table 2). This is in contrast to other obligate intracellular parasites have relatively small, repeat free genomes (WOOLFIT et al. 2013). Most of the elements found in the wInn genome are also shared with the host, D. innubila (HILL et al. 2019), suggesting that inefficient selection has not allowed these TEs to be maintained for extensive periods of time, but instead these elements are recent acquisitions (YOSHIYAMA et al. 2001), possibly vectored by prophage transmission. It is possible that eventually these TEs will be shed from the $w$ Inn genome and similar repeat families have been acquired in the past and eventually gone extinct and removed from the genome, in a cyclical fashion (MARUYAMA AND HARTL 1991; LOHE et al. 1995). Previous work in wMelPop suggests that reduced selection allowed repeats to accumulate in the Wolbachia genomes (WOOLFIT et al. 2013). This could also be the case for wInn, allowing these families to be maintained in the genome for extended periods of time, as opposed to removed immediately. The lack of similarity between $w$ Inn and $w$ MelPop repeats suggests that mobile elements have not been maintained since the common ancestor of these two Wolbachia strains, and that turnover is much more frequent.

Beyond just genome content, we analyzed the evolutionary dynamics of the genes in $w$ Inn. The overall finding, in line with what is known about phage biology, is that prophage and Octomom genes are

574 indeed rapidly evolving along all Wolbachia branches analyzed, although not significantly so. There was 575 also a trend of potentially more rapid evolution of DNA metabolism genes in $w$ Inn (Figure 2). Specifically, 576 the genes with the strongest signal were involved in DNA repair, DNA binding, host integration, and 577 recombination (Supplementary Table 3). This could relate to what may be an unusual amount of horizontal 578 gene transfer or high number of repetitive elements in this system, as DNA metabolism genes would aid in 579 these dynamics. Looking more specifically at the cifA/B CI genes and the wmk male-killing gene candidate, 580 we find that they individually also show evidence of rapid evolution across the phylogeny, although they are not more rapidly evolving than the rest of the prophage region (Figure 2). These are putatively or 
empirically demonstrated key genes in parasitizing host reproduction and may be in an adaptive arms race with the host (BECKMANN et al. 2017; LEPAGE et al. 2017; LINDSEY et al. 2018; PERLMUTTER et al. 2019), an idea that is supported by the rapid evolution.

Regarding the Octomom regions, we find that these genes are rapidly evolving across all the Wolbachia genomes in the clade examined, as expected, not just in the male-killing Wolbachia (Figure 2).

587 The rapid evolution of these genes may indicate they are frequently involved in host-pathogen interactions 588 as suggested elsewhere (CHROSTEK AND TEIXEIRA 2015), and as is seen with immune genes and other 589 genes involved in interspecies arms races (DAWKINS AND KREBS 1979; SACKTON et al. 2007; OBBARD et 590 al. 2009; PALMER et al. 2018). Indeed, previous studies have found an association between Octomom copy 591 number and increased titer (CHROSTEK AND TEIXEIRA 2015), as we see here (Supplementary Figure 5). 592 Additionally, while prophage may be able to excise themselves for transfer, Octomom may utilize transposable elements for horizontal transfer (CHROSTEK AND TEIXEIRA 2015). In line with this, the Octomom genes are fragmented across the genome rather than remaining in full cassettes, and the horizontal transfer of transposable elements is more and more frequently being found to occur between species (PECCOUd et al. 2017; Hill AND BetANCOURT 2018; Wallau et al. 2018). Given the presence of Varroa repeat elements in the $w$ Inn genome, this also provides a suitable vector organism to transfer these genes between Wolbachia types. When looking for evidence of recent horizontal transfer to wInn more generally (after divergence from $w \mathrm{Ha}$ and $w \mathrm{Ri}$ ), 13 genes were identified as potentially horizontally transferred to wInn. Of these, 7 were prophage or Octomom genes (Figure 3), supporting all findings so far indicating particularly rapid evolution and frequent transfer of prophage and Octomom genes.

Finally, we compared the inheritance of mitochondria and wInn in D. innubila populations, and find there is overall geographic structure in both cases (Figure 4) (HILL AND UNCKLESS 2020b; HILL AND UNCKLESS 2020a). However, we also find some discordance in inheritance in the Wolbachia prophage region, likely due to the activity of the prophage (Figure 5) resulting in extensive horizontal gene transfer of this region (Figure 4, Table 1, Supplementary Figure 4). When examining the phylogeny of wInn genomes, we find some Prescott lines are grouped within Chiricahua lines, potentially driven by this horizontal gene transfer. Alternatively, since the populations are recently established (HILL AND UNCKLESS 2020b; HILL AND UNCKLESS 2020a), it may simply indicate that these lines and mitotypes differentially

610 segregated into these populations upon establishment and that the types dominant in Chiricahuas are rarer 611 in Prescott.

\section{Conclusion}

Here, we provide the first genome description of wInn of $D$. innubila and use population genomic analyses to understand the evolutionary dynamics of this symbiont. We show evidence of rapid evolution, particularly among prophage and Octomom regions. We also demonstrate that this system is potentially 
experiencing high rates of horizontal transfer of genes, phages, or entire symbionts. This may occur not only within or between $D$. innubila populations, but across divergent mite and ant species as well. These dynamics may contribute to the success of Wolbachia symbionts in these populations and may reflect a broader strategy for survival and adaptation in diverse Wolbachia around the globe.

\section{Acknowledgements}

This manuscript was conceived and written thanks to suggestions and feedback from the Unckless lab at the University of Kansas. Collections were completed with assistance from Todd Schlenke at the University of Arizona, and the Southwest Research Station. The initial wInn sample used for genome sequencing was graciously provided by John Jaenike. We thank Brittny Smith and Jenny Hackett at the KU CMADP Genome Sequencing Core (NIH Grant P20 GM103638) and K-INBRE Bioinformatics Core for assistance in genome isolation, library preparation, sequencing and computational resources.

\section{Funding}

This work was supported by a K-INBRE postdoctoral grant to TH (NIH Grant P20 GM103418). This work was also funded by NIH Grants R00 GM114714 and R01 AI139154 to RLU.

\section{Data Availability}

All sequencing data used in this study is available on the NCBI SRA under the project accession:

PRJNA524688. Additional data regarding D. innubila population genomics is available in the following FigShare folder: https://figshare.com/account/home\#/projects/87662 and the following DataDryad submission: https://datadryad.org/stash/share/wvfmDL39pdYrVUcgDFAfI33BOJu3KCJWuJyj-0M-qgA.

\section{Author contributions}

TH devised analyses, performed genome assembly, performed analyses and wrote the manuscript, JIP devised analyses and wrote the manuscript, RLU devised analyses and wrote the manuscript.

\section{Ethics approval and consent to participate}

Not applicable.

\section{Consent for publication}

Not applicable.

\section{Conflicts of Interest}

The authors declare no conflicts of interest

\section{References}

Altschul, S. F., W. Gish, W. Miller, E. W. Myers and D. J. Lipman, 1990 Basic local alignment search tool. Journal of Molecular Biology 215: 403-410. 
681

682

683

684

685

686

687

688

689

690

691

692

693

694

695

Baldo, L., N. A. Ayoub, C. Y. Hayashi, J. A. Russell, J. K. Stahlhut et al., 2008 Insight into the routes of Wolbachia invasion: high levels of horizontal transfer in the spider genus Agelenopsis revealed by Wolbachia strain and mitochondrial DNA diversity. Molecular Ecology 17: 557-569.

Bankevich, A., S. Nurk, D. Antipov, A. A. Gurevich, M. Dvorkin et al., 2012 SPAdes: A new genome assembly algorithm and its applications to single-cell sequencing. Journal of computational biology 19: 455-477.

Beckmann, J. F., J. A. Ronau and M. Hochstrasser, 2017 A Wolbachia deubiquitylating enzyme induces cytoplasmic incompatibility. Nature Microbiology 2: 17007.

Beckmann, J. F., G. D. Sharma, L. Mendez, H. Chen and M. Hochstrasser, 2019 The Wolbachia cytoplasmic incompatibility enzyme CidB targets nuclear import and protamine-histone exchange factors. eLife 8: 1-23.

Berec, L., D. Maxin and V. Bernhauerova, 2016 Male-killing bacteria as agents of insect pest control. Journal of Applied Ecology.

Bolton, B., G. Alpert, P. S. Ward and P. Naskrecki, 2006 Bolton's Catalogue of Ants of the World. Cambridge: Harvard.

Bordenstein, S. R., and S. R. Bordenstein, 2016 Eukaryotic association module in phage WO genomes from Wolbachia. Nature Communications 7: 13155.

Bouchon, D., T. Rigaud and P. Juchault, 1998 Evidence for widespread Wolbachia infection in isopod crustaceans: molecular identification and host feminization. Proc Biol Sci 265: 1081-1090.

Brown, A. H. D., 1970 The estimation of Wright's fixation index from genotypic frequencies. Genetica 41: 399-406.

Brown, A. N., and V. K. Lloyd, 2015 Evidence for horizontal transfer of Wolbachia by a Drosophila mite. Exp Appl Acarol 66: 301-311.

Chakraborty, M., R. Zhao, X. Zhang, S. Kalsow and J. J. Emerson, 2017 Extensive hidden genetic variation shapes the structure of functional elements in Drosophila. Doi.Org 50: 114967.

Chrostek, E., and L. Teixeira, 2015 Mutualism breakdown by amplification of Wolbachia genes. PLoS Biology 13: e1002065.

Cooper, B. S., D. Vanderpool, W. R. Conner, D. R. Matute and M. Turelli, 2019 Wolbachia acquisition by Drosophila yakuba-clade hosts and transfer of incompatibility loci between distantly related Wolbachia. Genetics 212: 1399-1419.

Danecek, P., A. Auton, G. Abecasis, C. A. Albers, E. Banks et al., 2011 The variant call format and VCFtools. Bioinformatics 27: 2156-2158.

Dawkins, R., and J. R. Krebs, 1979 Arms race between and within species. Proceedings of the Royal Society of London B: Biological Sciences 205: 489-511.

DePristo, M. A., E. Banks, R. Poplin, K. V. Garimella, J. R. Maguire et al., 2011 A framework for variation discovery and genotyping using next-generation DNA sequencing data. Nature genetics 43: 491-498.

Duarte, E. H., A. Carvalho, S. Lopez-Madrigal and L. Teixeira, 2020 Regulation of Wolbachia proliferation by the amplification and deletion of an addictive genomic island. bioRxiv.

Duplouy, A., I. Iturbe-Ormaetxe, S. A. Beatson, J. M. Szubert, J. C. Brownlie et al., 2013 Draft genome sequence of the male-killing Wolbachia strain $w$ Boll reveals recent horizontal gene transfers from diverse sources. BMC Genomics 14: 1-13.

Dutta, C., and A. Pan, 2002 Horizontal gene transfer and bacterial diversity. J. Biosci. 27: 27-33.

Dyer, K. A., and J. Jaenike, 2004 Evolutionarily stable infection by a male-killing endosymbiont in Drosophila innubila: molecular evidence from the host and parasite genomes. Genetics 168: 1443-1455.

Dyer, K. a., and J. Jaenike, 2005 Evolutionary dynamics of a spatially structured host-parasite association: Drosophila innubila and male-killing Wolbachia. Evolution; international journal of organic evolution 59: 1518-1528. 
Dyer, K. A., M. S. Minhas and J. Jaenike, 2005 Expression and modulation of embryonic male-killing in Drosophila innubila: opportunities for multilevel selection. Evolution; international journal of organic evolution 59: 838-848.

Dyson, E., M. Kamath and G. Hurst, 2002 Wolbachia infection associated with all-female broods in Hypolimnas bolina (Lepidoptera: Nymphalidae): evidence for horizontal transmission of a butterfly male killer. Heredity 88: 166-171.

Foster, J., M. Ganatra, I. Kamal, J. Ware, K. Makarova et al., 2005 The Wolbachia genome of Brugia malayi: endosymbiont evolution within a human pathogenic nematode. PLoS Biol 3: e121.

Francoeur, A., 1973 Révision taxonomique des éspèces nearctique du group fusca, genre Formica (Formicidae: Hymenoptera). Mémoires de la Société entomologique du Québec 3: 1-316.

Fujii, Y., D. Kageyama, S. Hoshizaki, H. Ishikawa and T. Sasaki, 2001 Transfection of Wolbachia in Lepidoptera: the feminizer of the adzuki bean borer Ostrinia scapulalis causes male killing in the Mediterranean flour moth Ephestia kuehniella. Proceedings of the Royal Society of London. Series B: Biological Sciences 268: 855-859.

Gerth, M., M.-T. Gansauge, A. Weigert and C. Bleidorn, 2014 Phylogenomic analyses uncover origin and spread of the Wolbachia pandemic. Nature communications 5: 1-7.

Guindon, S., J.-F. Dufayard, V. Lefort, M. Anisimova, W. Hordijk et al., 2010 New algorithms and methods to estimate maximum-likelihood phylogenies: assessing the performance of PhyML 3.0. Systematic biology 59: 307-321.

Haine, E. R., N. J. Pickup and J. M. Cook, 2005 Horizontal transmission of Wolbachia in a Drosophila community. Ecological Entomology 30: 464-472.

Hedges, L. M., J. C. Brownlie, S. L. Neill and K. N. Johnson, 2008 Wolbachia and Virus Protection in Insects. Science 322: 702.

Hertig, M., and S. B. Wolbach, 1924 Studies on Rickettsia-Like Micro-Organisms in Insects. J Med Res 44: 329-374.327.

Hill, T., and A. Betancourt, 2018 Extensive horizontal exchange of transposable elements in the Drosophila pseudoobscura group. Mobile DNA 20: 1-14.

Hill, T., B. Koseva and R. L. Unckless, 2019 The genome of Drosophila innubila reveals lineage-specific patterns of selection in immune genes. Molecular Biology and Evolution 36: 1405-1417.

Hill, T., and R. Unckless, 2020a Adaptation, ancestral variation and gene flow in a 'Sky Island' Drosophila species. Molecular Ecology: 1-35.

Hill, T., and R. Unckless, 2020b Recurrent evolution of high virulence in isolated populations of a DNA virus. eLife 9: e58931.

Hornett, E. A., S. Charlat, A. M. Duplouy, N. Davies, G. K. Roderick et al., 2006 Evolution of male-killer suppression in a natural population. PLoS Biol 4: e283.

Houck, M. A., J. B. Clark, K. R. Peterson and M. G. Kidwell, 1993 Possible horizontal transfer of Drosophila genes by the mite Protolaelaps regalis. Science 253: 1125-1129.

Hughes, G. L., and J. L. Rasgon, 2014 Transinfection: a method to investigate Wolbachia-host interactions and control arthropod-borne disease. Insect Mol Biol 23: 141-151.

Hurst, G. D., and C. L. Frost, 2015 Reproductive parasitism: maternally inherited symbionts in a biparental world. Cold Spring Harbor perspectives in biology 7: a017699.

Hurst, G. D. D., F. M. Jiggins, J. Hinrich Graf von der Schulenburg, D. Bertrand, S. A. West et al., 1999 Male-killing Wolbachia in two species of insect. Proceedings of the Royal Society of London. Series B: Biological Sciences 266: 735-740.

Hurst, G. D. D., A. P. Johnson, J. H. G. v. d. Schulenburg and Y. Fuyama, 2000 Male-Killing Wolbachia in Drosophila: A Temperature-Sensitive Trait With a Threshold Bacterial Density. Genetics 156: 699.

Ilinsky, Y., 2013 Coevolution of Drosophila melanogaster mtDNA and Wolbachia Genotypes. PLoS ONE 8: e54373.

Jaenike, J., 2007 Spontaneous emergence of a new Wolbachia phenotype. Evolution 61: 2244-2252. 
Jaenike, J., and K. A. Dyer, 2008 No resistance to male-killing Wolbachia after thousands of years of infection. Journal of Evolutionary Biology 21: 1570-1577.

Jaenike, J., K. A. Dyer and L. K. Reed, 2003 Within-population structure of competition and the dynamics of male-killing Wolbachia. Evolutionary Ecology Research 5: 1023-1036.

Jain, M., H. E. Olsen, B. Paten and M. Akeson, 2016 Erratum to: The Oxford Nanopore MinION: Delivery of nanopore sequencing to the genomics community. Genome Biology 17: 1-11.

Jiggins, F. M., G. D. D. Hurst and M. E. N. Majerus, 2000 Sex-ratio-distorting Wolbachia causes sex-role reversal in its butterfly host. Proceedings of the Royal Society of London. Series B: Biological Sciences 267: 69-73.

Kageyama, D., G. Nishimura, S. Hoshizaki and Y. Ishikawa, 2002 Feminizing Wolbachia in an insect, Ostrinia furnacalis (Lepidoptera: Crambidae). Heredity 88: 444-449.

Katoh, K., K. Misawa, K.-i. Kuma and T. Miyata, 2002 MAFFT: a novel method for rapid multiple sequence alignment based on fast Fourier transform. Nucleic acids research 30: 3059-3066.

Laidoudi, Y., A. Levasseur, H. Medkour, M. Maaloum, M. Ben Khedher et al., 2020 An Earliest Endosymbiont, Wolbachia massiliensis sp. nov., Strain PL13 from the Bed Bug (Cimex hemipterus), Type Strain of a New Supergroup T. Int J Mol Sci 21.

Lawrence, J., 1999 Gene transfer, speciation, and the evolution of bacterial genomes. Current opinion in microbiology 2: 519-523.

Lefoulon, E., T. Clark, F. Borveto, M. Perriat-Sanguinet, C. Moulia et al., 2020 Pseudoscorpion Wolbachia symbionts: Diversity and Evidence for a New Supergroup S. Research Square: 1-37.

LePage, D., and S. R. Bordenstein, 2013 Wolbachia: Can we save lives with a great pandemic? Trends Parasitol 29: 385-393.

LePage, D. P., J. A. Metcalf, S. R. Bordenstein, J. On, J. I. Perlmutter et al., 2017 Prophage WO genes recapitulate and enhance Wolbachia-induced cytoplasmic incompatibility. Nature 543: 243-247.

Li, H., and R. Durbin, 2009 Fast and accurate short read alignment with Burrows-Wheeler transform. Bioinformatics (Oxford, England) 25: 1754-1760.

Li, H., B. Handsaker, A. Wysoker, T. Fennell, J. Ruan et al., 2009 The sequence alignment/map format and SAMtools. Bioinformatics (Oxford, England) 25: 2078-2079.

Lindsey, A. R., D. W. Rice, S. R. Bordenstein, A. W. Brooks, S. R. Bordenstein et al., 2018 Evolutionary genetics of cytoplasmic incompatibility genes cifA and cifB in prophage WO of Wolbachia. Genome biology and evolution 10: 434-451.

Lohe, A. R., E. N. Moriyama, D. A. Lidholm and D. L. Hartl, 1995 Horizontal transmission, vertical inactivation, and stochastic loss of mariner-like transposable elements. Molecular biology and evolution 12: 62-72.

Löytynoja, A., 2014 Phylogeny-aware alignment with PRANK, pp. 155-170 in Multiple Sequence Alignment Methods, edited by D. J. Russell. Humana Press, Totowa, NJ.

Mains, J. W., P. H. Kelly, K. L. Dobson, W. D. Petrie and S. L. Dobson, 2019 Localized Control of Aedes aegypti (Diptera: Culicidae) in Miami, FL, via Inundative Releases of Wolbachia-Infected Male Mosquitoes. Journal of Medical Entomology 56: 1296-1303.

Majerus, T. M., and M. E. Majerus, 2010 Intergenomic arms races: detection of a nuclear rescue gene of male-killing in a ladybird. PLoS Pathog 6: e1000987.

Maruyama, K., and D. L. Hartl, 1991 Evolution of the transposable element Mariner in Drosophila Species. Genetics Society of America 128: 319-329.

McCutcheon, J. P., and N. A. Moran, 2012 Extreme genome reduction in symbiotic bacteria. Nature Reviews Microbiology 10: 13-26.

McKenna, A., M. Hanna, E. Banks, A. Sivachenko, K. Cibulskis et al., 2010 The Genome Analysis Toolkit: A MapReduce framework for analyzing next-generation DNA sequencing data. Proceedings of the International Conference on Intellectual Capital, Knowledge Management \& Organizational Learning 20: 1297-1303. 
Metcalf, J. A., M. Jo, S. R. Bordenstein, J. Jaenike and S. R. Bordenstein, 2014 Recent genome reduction of Wolbachia in Drosophila recens targets phage WO and narrows candidates for reproductive parasitism. PeerJ 2: e529.

Moran, N. A., 2002 Microbial minimalism: genome reduction in bacterial pathogens. Cell 108: 583-586.

Narasimhan, V., P. Danecek, A. Scally, Y. Xue, C. Tyler-Smith et al., 2016 BCFtools/RoH: A hidden Markov model approach for detecting autozygosity from next-generation sequencing data. Bioinformatics 32: 1749-1751.

O'Neill, S. L., P. A. Ryan, A. P. Turley, G. Wilson, K. Retzki et al., 2018 Scaled deployment of Wolbachia to protect the community from dengue and other Aedes transmitted arboviruses. Gates open research 2.

Obbard, D. J., J. J. Welch, K. W. Kim and F. M. Jiggins, 2009 Quantifying adaptive evolution in the Drosophila immune system. PLoS Genetics 5: e1000698.

Palmer, W. H., J. D. Hadfield and D. J. Obbard, 2018 RNA Interference Pathways Display High Rates of Adaptive Protein Evolution in Multiple Invertebrates. Genetics: genetics.300567.302017.

Paradis, E., J. Claude and K. Strimmer, 2004 APE: analyses of phylogenetics and evolution in R language. Bioinformatics 20: 289-290.

Peccoud, J., V. Loiseau, R. Cordaux and C. Gilbert, 2017 Massive horizontal transfer of transposable elements in insects. Proceedings of the National Academy of Sciences 114: 4721-4726.

Perlmutter, J. I., S. R. Bordenstein, R. L. Unckless, D. P. LePage, J. A. Metcalf et al., 2019 The phage gene wmk is a candidate for male killing by a bacterial endosymbiont. PLoS pathogens 15: e1007936.

Pertea, G., 2011 gffread, pp., https://github.com/gpertea/gffread.

Pruitt, K. D., T. Tatusova and D. R. Maglott, 2005 NCBI Reference Sequence (RefSeq): a curated nonredundant sequence database of genomes, transcripts and proteins. Nucleic Acids Res 33: D501504.

Riegler, M., M. Sidhu, W. J. Miller and S. L. O'Neill, 2005 Evidence for a global Wolbachia replacement in Drosophila melanogaster. Current biology 15: 1428-1433.

Rosenkranz, P., P. Aumeier and B. Ziegelmann, 2010 Biology and control of Varroa destructor. Journal of invertebrate pathology 103: S96-S119.

Ross, P. A., M. Turelli and A. A. Hoffmann, 2019 Evolutionary Ecology of Wolbachia Releases for Disease Control. Annual Review of Genetics 53: 93-116.

Russell, J. E., and R. Stouthamer, 2011 The genetics and evolution of obligate reproductive parasitism in Trichogramma pretiosum infected with parthenogenesis-inducing Wolbachia. Heredity 106: 5867.

Sackton, T. B., B. P. Lazzaro, T. A. Schlenke, J. D. Evans, D. Hultmark et al., 2007 Dynamic evolution of the innate immune system in Drosophila. Nature Genetics 39: 1461-1468.

Sanaei, E., S. Charlat and J. Engelstädter, 2020 Wolbachia host shifts: routes, mechanisms, constraints and evolutionary consequences. Biological Reviews.

Sasaki, T., T. Kubo and H. Ishikawa, 2002 Interspecific Transfer of Wolbachia Between Two Lepidopteran Insects Expressing Cytoplasmic Incompatibility: A Wolbachia Variant Naturally Infecting Cadra cautella Causes Male Killing in Ephestia kuehniella. Genetics 162: 1313-1319.

Seemann, T., 2014 Prokka: rapid prokaryotic genome annotation. Bioinformatics 30: 2068-2069.

Serbus, L. R., and W. Sullivan, 2007 A cellular basis for Wolbachia recruitment to the host germline. PLoS Pathogens 3: e190.

Sheeley, S. L., and B. F. McAllister, 2009 Mobile male-killer: similar Wolbachia strains kill males of divergent Drosophila hosts. Heredity 102: 286-292.

Simão, F. A., R. M. Waterhouse, P. Ioannidis, E. V. Kriventseva and E. M. Zdobnov, 2015 BUSCO: Assessing genome assembly and annotation completeness with single-copy orthologs. Bioinformatics 31: 3210-3212. 
Sinkins, S. P., H. R. Braig and S. L. O'Neill, 1995 Wolbachia superinfections and the expression of cytoplasmic incompatibility. Proceedings of the Royal Society of London. Series B: Biological Sciences 261: 325-330.

Smit, A. F. A., and R. Hubley, 2008 RepeatModeler Open-1.0.

Smit, A. F. A., and R. Hubley, 2013-2015 RepeatMasker Open-4.0, pp. RepeatMasker.

Taylor, M. J., S. R. Bordenstein and B. Slatko, 2018 Microbe Profile: Wolbachia: a sex selector, a viral protector and a target to treat filarial nematodes. Microbiology 164: 1345-1347.

Team, R. C., 2013 R: A Language and Environment for Statistical Computing, pp. R Foundation for Statistical Computing, Vienna, Austria.

Teixeira, L., A. Ferreira and M. Ashburner, 2008 The bacterial symbiont Wolbachia induces resistance to RNA viral infections in Drosophila melanogaster. PLoS biology 6: e2.

Turelli, M., B. S. Cooper, K. M. Richardson, P. S. Ginsberg, B. Peckenpaugh et al., 2018 Rapid Global Spread of wRi-like Wolbachia across Multiple Drosophila. Current Biology 28: 963 - 971.

Turelli, M., and A. A. Hoffman, 1991 Rapid spread of an inherited incompatibility factor in California Drosophila. Nature 353: 440-442.

Unckless, R. L., and J. Jaenike, 2012 Maintenance of a Male-Killing Wolbachia in Drosophila innubila By Male-Killing Dependent and Male-Killing Independent Mechanisms. Evolution 66: 678-689.

Vaser, R., I. Sovic, N. Nagarajan and M. Sikic, 2017 Fast and accurate de novo genome assembly from long uncorrected reads. Genome Res 27: 737-746.

Vavre, F., F. Fleury, D. Lepetit, P. Fouillet and M. Boulétreau, 1999 Phylogenetic evidence for horizontal transmission of Wolbachia in host-parasitoid associations. Molecular Biology and Evolution 16: 1711-1723.

Walker, B. J., T. Abeel, T. Shea, M. Priest, A. Abouelliel et al., 2014 Pilon: An integrated tool for comprehensive microbial variant detection and genome assembly improvement. PLoS ONE 9.

Wallau, G. L., P. Capy, E. Loreto, A. L. Rouzic and A. Hua-Van, 2016 VHICA, a New method to discriminate between vertical and horizontal transposon transfer: Application to the mariner family with in Drosophila. Molecular Biology and Evolution 33: 1094-1109.

Wallau, G. L., C. Vieira and É. L. S. Loreto, 2018 Genetic exchange in eukaryotes through horizontal transfer: Connected by the mobilome. Mobile DNA 9: 1-16.

Wang, G. H., B. F. Sun, T. L. Xiong, Y. K. Wang, K. E. Murfin et al., 2016 Bacteriophage WO Can Mediate Horizontal Gene Transfer in Endosymbiotic Wolbachia Genomes. Frontiers in Microbiology 7: 1-16.

Weinert, L. A., E. V. Araujo-Jnr, M. Z. Ahmed and J. J. Welch, 2015 The incidence of bacterial endosymbionts in terrestrial arthropods. Proceedings of the Royal Society B: Biological Sciences 282: 20150249.

Werren, J. H., L. Baldo and M. E. Clark, 2008 Wolbachia: master manipulators of invertebrate biology. Nat Rev Microbiol 6: 741-751.

Wicker, T., F. Sabot, A. Hua-Van, J. L. Bennetzen, P. Capy et al., 2007 A unified classification system for eukaryotic transposable elements. Nature reviews. Genetics 8: 973-982.

Woolfit, M., I. Iturbe-Ormaetxe, J. C. Brownlie, T. Walker, M. Riegler et al., 2013 Genomic evolution of the pathogenic Wolbachia strain, wMelPop. Genome Biol Evol 5: 2189-2204.

Yang, Z., 2007 PAML 4: Phylogenetic analysis by maximum likelihood. Molecular Biology and Evolution 24: 1586-1591.

Yen, J. H., and A. R. Barr, 1971 New hypothesis of the cause of cytoplasmic incompatibility in Culex pipiens L. Nature 232: 657-658.

Yoshiyama, M., Z. Tu, Y. Kainoh, H. Honda, T. Shono et al., 2001 Possible horizontal transfer of a transposable element from host to parasitoid. Molecular Biology and Evolution 18: 1952-1958.

Zabalou, S., M. Riegler, M. Theodorakopoulou, C. Stauffer, C. Savakis et al., 2004 Wolbachia-induced cytoplasmic incompatibility as a means for insect pest population control. Proceedings of the National Academy of Sciences of the United States of America 101 15042-15045. 

913 gene.

914 Supplementary Table 5: Gene orthologs identified in each non- $w$ Inn genome analysed, when looking for 915 discordance from the whole species phylogeny. Gene annotation for each genome is given for each column. 916 When the ortholog is absent from a genome it is labelled as NA.

917 Supplementary Table 6: $F_{\text {ST }}$ enrichments for functional categories across the wInn genome using a 918 generalized linear model.

Zhou, W., F. Rousset and S. O'Neill, 1998 Phylogeny and PCR-based classication of Wolbachia strains using wsp gene sequences. Proc. R. Soc. 265: 509-515.

Zimin, A. V., G. Marcais, D. Puiu, M. Roberts, S. L. Salzberg et al., 2013 The MaSuRCA genome assembler. Bioinformatics 29: 2669-2677.

Zug, R., and P. Hammerstein, 2012 Still a host of hosts for Wolbachia: analysis of recent data suggests that $40 \%$ of terrestrial arthropod species are infected. PLoS One 7: e38544.

\section{Supplementary Tables and Figures}

Supplementary Table 1: Data (genomes and next generation sequencing information) used in this study. When applicable the number of sequences/reads and the proportion of these that are Wolbachia are shown in the table. SRA accession numbers are included for both short reads and genomes.

Supplementary Table 2: Genes annotated in the $w$ Inn genome in GFF format, with their ID and $w$ Mel ortholog when applicable. GFF also includes locations of transposable element insertions and short simple repeats.

Supplementary Table 3: dN/dS estimations between $w$ Inn, $w$ Ha and $w$ Ri. Functional categories of genes are also noted in the table. Table includes both the non-synonymous ( $\mathrm{dN}$ ) and synonymous (dS) estimations across the total phylogeny of $w$ Inn- $w \mathrm{Ha}-w \mathrm{Ri}$ and on each branch of the phylogeny.

Supplementary Table 4: Table of VHICA output and divergence estimates. For each gene (using the wInn gene name), we include the measure of effective codon usage (CUB), the dS pairwise between $w \mathrm{Ha}, w \operatorname{Inn}$ and $w \mathrm{Ri}$. If the gene has elevated $\mathrm{dS}$, we note this. Table also include the gene ontology category of the 
919 Supplementary Figure 1. Phylogeny of Wolbachia genomes used in this survey. A subset of this

920 phylogeny is used in Figure 1B. Nodes with 100 bootstrap support are not labelled, nodes with fewer than

921100 bootstraps support are labelled.

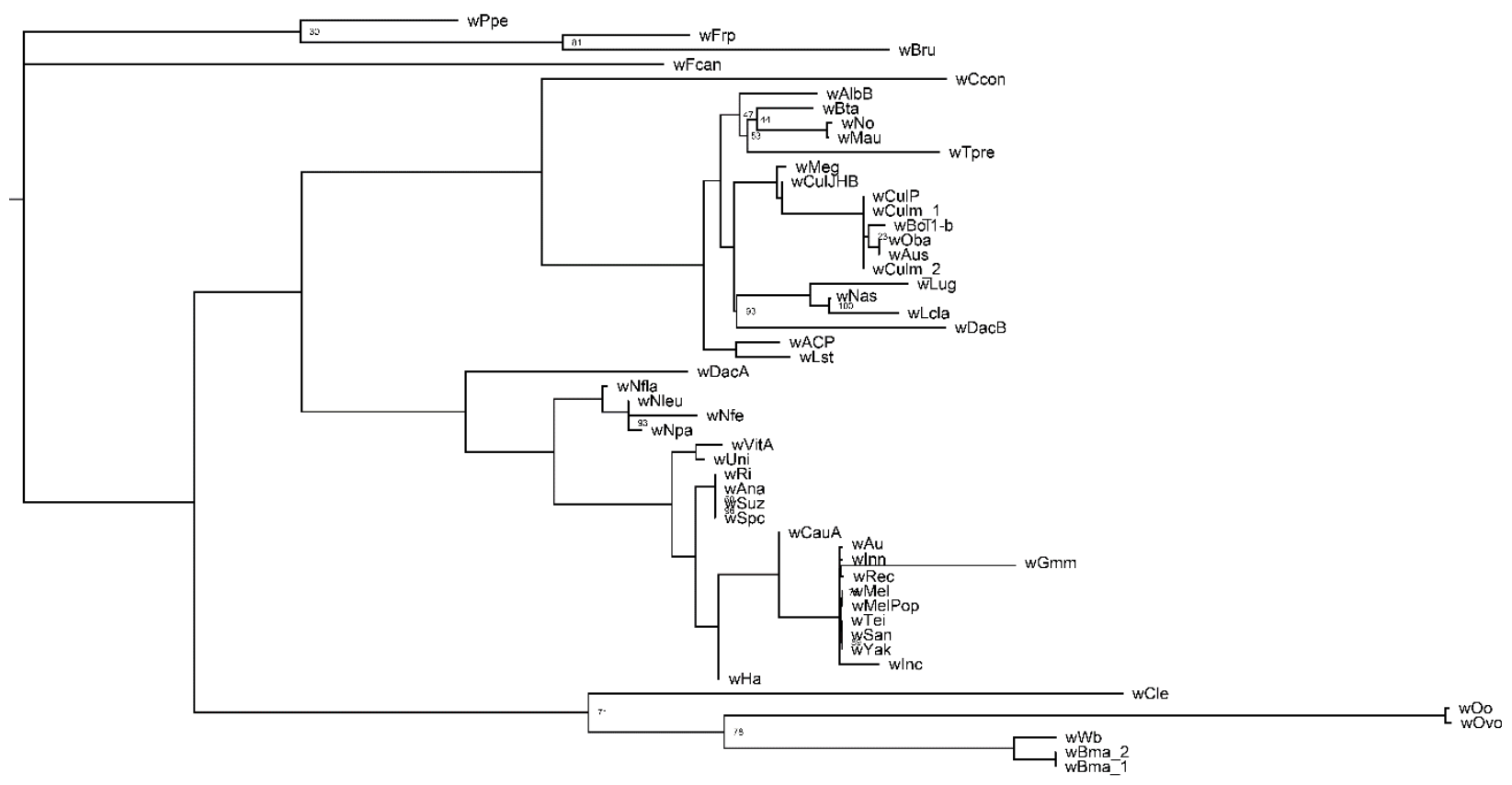

923 Supplementary Figure 2. Transposable element insertions in three windows across the genome, separated

924 by full and partial insertions, colored by TE order. Only regions of the genome containing transposable

925 elements are shown, to avoid plotting large gapped regions.

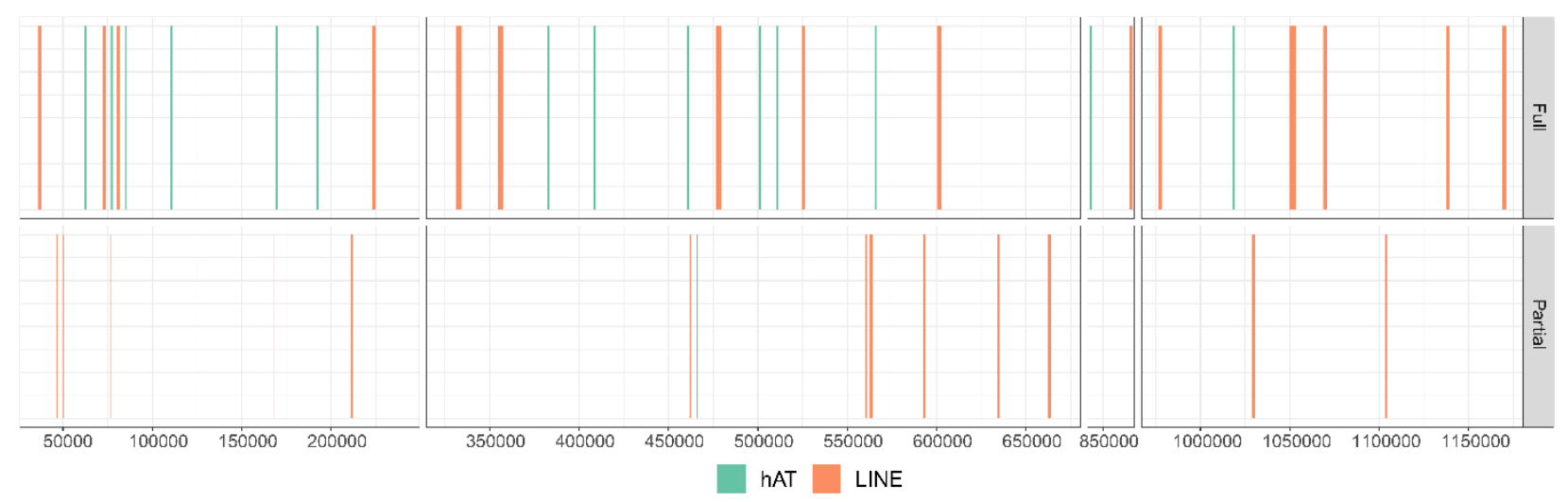


927 Supplementary Figure 3: Maximum-Likelihood phylogenies of A. Non-prophage Wolbachia genes. B.

928 the D. innubila mitochondria. Branches are colored by the population the tip sample belongs to. Nodes

929 connected to different colored branches are colored by the branch with the most tips.

A



B

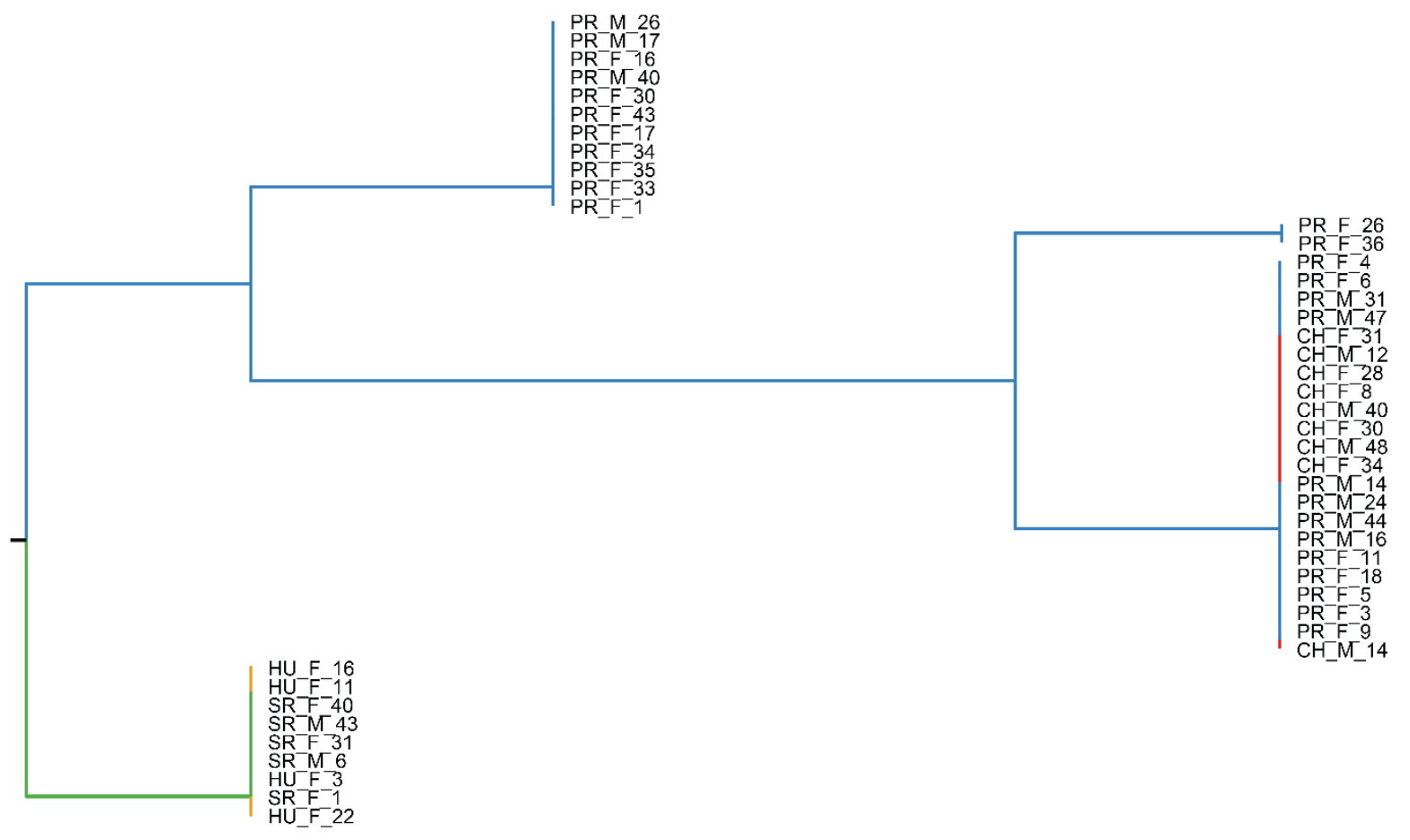


bioRxiv preprint doi: https://doi.org/10.1101/2020.11.16.385294; this version posted November 17,2020 . The copyright holder for this preprint (which was not certified by peer review) is the author/funder, who has granted bioRxiv a license to display the preprint in perpetuity. It is made available under aCC-BY-NC 4.0 International license.

931 Supplementary Figure 4: Proportion of SNPs in windows (10kbp windows, sliding 2kbp) across the 932 wInn genome that show discordant inheritance compared to mitochondrial SNPs. Blue shaded blocks

933 highlight the start and end of phage WO portions of the genome. For simplicity we have also shaded the 934 regions between phage blocks of the same type (Figure 1).

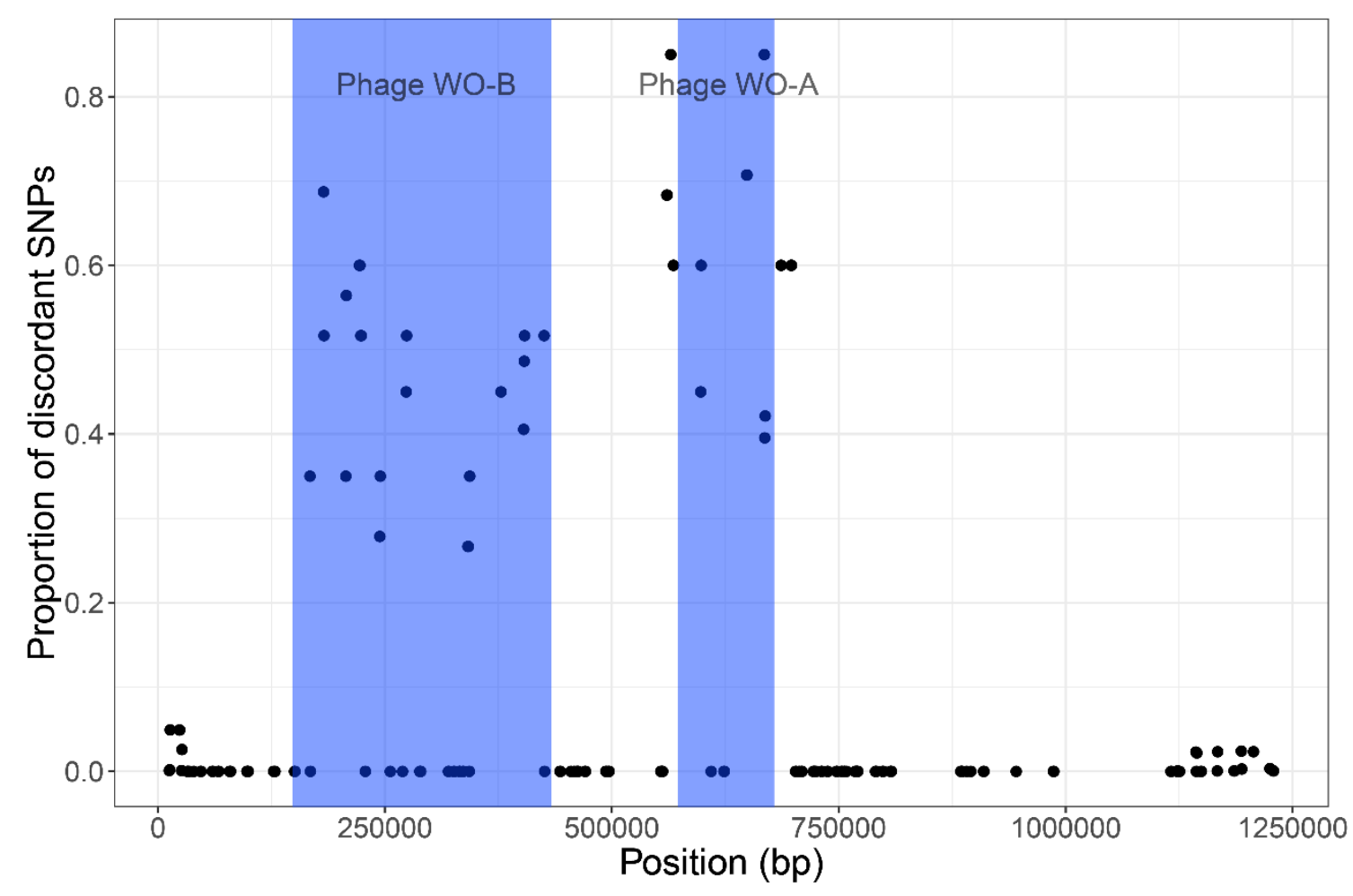

